\title{
SHALLOW WATER VISCOUS FLOWS FOR ARBITRARY TOPOGRAPHY*
}

\author{
MARC BOUTOUNET ${ }^{\dagger}$, LAURENT CHUPIN ${ }^{\ddagger}$, PASCAL NOBLE ${ }^{\S}$, AND JEAN PAUL VILA
}

\begin{abstract}
In this paper, we obtain new models for gravity driven shallow water laminar flows in several space dimensions over a general topography. These models are derived from the incompressible Navier-Stokes equations with no-slip condition at the bottom and include capillary effects. No particular assumption is made on the size of the viscosity and on the variations of the slope. The equations are written for an arbitrary parametrization of the bottom, and an explicit formulation is given in the orthogonal courvilinear coordinates setting and for a particular parametrization so-called "steepest descent" curvilinear coordinates.
\end{abstract}

Key words. shallow water, arbitrary topography, capillary effects

AMS subject classifications. 76B15, 35Q35, 86A05

\section{Introduction}

Mathematical models and numerical simulations for the flow of a relatively thin layer of fluid under the influence of gravity over a complex relief have important applications in natural processes such as ocean modeling, flows in rivers and coastal areas, debris avalanches or industrial processes such as coating flows with applications ranging from a single decorative layer on packaging to multiple-layer coatings on photographic film. See the review paper on thin fluid dynamics by Oron, Davis and Bankoff [8] and bibliography therein for further references.

Herein, we consider the slow motion of a thin liquid layer over an arbitrary topography. The fluid is assumed to be incompressible and Newtonian. It is submitted to capillary forces at the free surface and a no-slip condition is assumed at the bottom surface. Our particular interest is to take into account as much as possible the influence of the topography in the flow equations. The dynamics are now well understood in the flat case. For completeness, let us summarize recent results obtained in the modeling of flows down a flat bottom. Given the fact that three dimensional NavierStokes equations are difficult to treat both analytically and numerically, in particular if the boundary is free, it is important to obtain reduced models that are able to capture the relevant features, but are mathematically more manageable. Modeling thin film flows down an inclined plane leads to a hierarchy of models. The first stage of approximation is the lubrication model: under a suitable scaling, the fluid speed and pressure are determined by the local fluid height and its dervatives. In that case we obtain a model for the local fluid height in the form

$$
\partial_{t} h=G\left(h, \partial_{x^{m}} h\right),
$$

${ }^{*}$ Received: June 26, 2007; accepted (in revised version): October 26, 2007. Communicated by Francois Bouchut.

†ONERA, 2 Avenue E. Belin, 31055 Toulouse Cedex, France (boutounet@insa-toulouse.fr).

¥Pôle de Mathématiques, Bâtiment Léonard de Vinci, 21, avenue Jean Capelle, 69621 Villeurbanne cedex, France (Laurent.Chupin@insa-lyon.fr).

$\S$ Université de Lyon, Université Lyon 1, CNRS, UMR 5208, Institut Camille Jordan, Batiment du Doyen Jean Braconnier, 43, blvd du 11 novembre 1918, F - 69622 Villeurbanne Cedex, France (noble@math.univ-lyon1.fr).

IInstitut de Mathématiques de Toulouse, CNRS, UMR 5219, INSA de Toulouse, 135 avenue de Rangueil, 31077 Toulouse Cedex 4-, France (vila@insa-toulouse.fr). 
where $G$ involves various algebraic powers and differentiation orders of $h$. This type of equation is obtained by means of asymptotic expansions in power of $\varepsilon$, usually called the film parameter or aspect ratio between the width of the layer and the typical length of the phenomena in the streamwise direction. The simplest model obtained in this way is Benney's Equation [12]. The simplification brought by this reduction has permitted a first study of the nonlinear development of waves using dynamical system theory [13]. Unfortunately, Benney's equation exhibits finite time singularities and is only valid for small amplitude waves.

In order to obtain models valid for thicker flows, one considers shallow-water-type flows: a specific shape for the velocity profile is assumed together with a hydrostatic field assumption. Averaging the streamwise momentum equation and the divergencefree condition, one obtains an evolution system for the local fluid height $h$ and the local flow rate $q=\int_{0}^{h} u(z) d z$. As a first approximation, one can assume that the velocity is constant along the fluid height: this approach is justified for shallow water flows of incompressible Newtonian fluids with negligible viscosity: see Perthame and co-authors [7] for a formal derivation from the Navier Stokes equations and numerical simulations of the resulting shallow water equations, Bouchut and co-authors $[1,2]$ for the case of non-flat bottoms. When the viscosity is not small and a no-slip condition at the bottom is assumed, we are in a regime where the fluid layer is entirely a boundary layer: for inclined planes, the stationary solutions are of Nusselt type with a parabolic profile of velocity and constant height. In that case, the first integral boundary layer model was derived by Shkadov [11], assuming that the flow remains close to the Nusselt flow. More recently, Ruyer-Quil and Manneville [5, 6] generalized this approach and even derived second order models by introducing a third variable $\tau$ that measures the departure of the wall shear from the shear predicted by the parabolic velocity profile. Up to order one, this variable is determined by the local fluid height $h$ and the local discharge rate $q$; this yields a refined version of the shallow water equations. The method of derivation is the following: the Navier-Stokes solutions are expanded along a basis of polynomials in the cross-stream variable with slowly varying coefficients, these coefficients being determined under specific rules of projections (collocation methods or Galerkin methods): this leads to a family of models that differs one from the other through different coefficients. In fact, this method generates a family of models, all accurate to the same order; the coefficients only depend on the basis chosen to expand the solutions and the projection rules. J.-P. Vila [14] has unified the formulation of the different models into a family of shallow-water-type models parametrized by free constants: under the shallow water scaling, Navier-Stokes solutions close to the Nusslet flow are expanded with respect to $\varepsilon$, the film parameter, and different characteristic numbers of the fluid (Reynolds, Froude, Weber numbers). The expansion is inserted into the exact average equations for the fluid height $h$ and the discharge rate $h v=\int_{0}^{h} u, u$ being the component of the fluid velocity in the streamwise direction. Dropping small terms to a fixed order in $\varepsilon$, a family of shallow water models in a closed form is obtained. A linear stability analysis of constant solutions is carried out and compared to the Orr-Sommerfeld stability equations for the full Navier-Stokes system in the limit of large wavelength in order to check the accuracy of the shallow water models.

When the topography is arbitrary, this approach has been generalized using the center manifold framework $[9,10]$ : in that case the computations are carried out in the neighborhood of the Nusselt parabolic flow. Using the center manifold reduction, Roberts and co-workers formally obtained lubrication models [9] and shallow water 
type models [10] under the hypothesis that the curvature of the underlying bottom surface is small. The models are formulated in a particular system of curvilinear coordinates, namely the Darboux coordinates, the two basis vectors tangent to the surface being pointed in the direction of maximal and minimal curvature. The Darboux system of coordinates has the disadvantage not to be defined at umbilic points, i.e., points where the principal curvatures coincide.

Whereas the situation is now well understood for the flat and almost flat case from the modeling point of view, and the mathematical theory has become settled now $[3,4]$, the situation is different for arbitrary topography: let us mention here the recent papers of Bouchut and co-authors $[1,2]$ on the derivation of one dimensional and multidimensional shallow water models from incompressible Euler equations (or Navier-Stokes equations with small viscous term) without any restriction on the topography and in the presence of a Coulomb friction term. One interesting aspect of the approach proposed by Bouchut and co-authors is that the derivation is valid for arbitrary topography and the formulation does not depend on the parametrization of the surface where the fluid flows. In this present paper, we consider the slow motion of a relatively thin layer of fluid over an arbitrary topography. The fluid is incompressible, Newtonian and the viscosity is not negligible. A no-slip condition is assumed at the bottom surface. For completeness, we also assume that it is submitted to capillary forces at the free surface. In this paper, we derive shallow water equations from the incompressible Navier-Stokes equations: as a byproduct of the analysis, we will also obtain lubrication models.

The paper is organized as follows. In Section 2, we formulate the Navier-Stokes equations and boundary conditions in a system of coordinates adapted to the geometry of the bottom surface; during this step, we do not make precise the parametrization of the underlying surface where the fluid flows. We also compute an exact evolution system for the physical height of the fluid $h$ and the average velocity, along the fluid height, parallel to the surface. This evolution system is not in a closed form and some modeling assumptions are necessary: classically, the pressure is supposed to be hydrostatic and the flow is quasi-stationary. In this paper, we follow the ideas introduced by J.-P. Vila in [14], and these assumptions will be a result of a dimensional analysis of the Navier Stokes system. In Section 3, we rescale the equations: the characteristic height of the fluid $H$ is supposed to be small compared to the characteristic wavelength $L$ of the flow variable in the streamwise direction. We introduce the "aspect ratio" $\varepsilon=\frac{H}{L} \ll 1$. In the asymptotic regime $\varepsilon \rightarrow 0$, we compute an expansion, with respect to $\varepsilon$, of the Navier-Stokes solutions close to a basic Nusselt type flow. As a byproduct of the dimensional analysis, we recover the classical modeling assumptions of the shallow water equations. Inserting the asymptotic expansions of Navier-Stokes solutions in the evolution system for the fluid height $h$ and the discharge rate $\int_{0}^{h} u$ and dropping "small terms", we obtain a shallow water system in a closed form.

In the scaling chosen, the fluid speed direction is mainly supported by the "steepest descent" direction. Thus it is natural to introduce a specific parametrization of the surface where the fluid flows, so called "steepest descent" curvilinear coordinates. The first vector of the basis is tangent to the surface and has the steepest descent direction, the third one is the upward normal to the surface and the second one is deduced from the others through a direct wedge product. In Section 4, we write the shallow water system in the "steepest descent" curvilinear coordinates and in a standard set of orthogonal curvilinear coordinates. Finally, we compute lubrication models from the shallow water system by eliminating the average velocity from the 


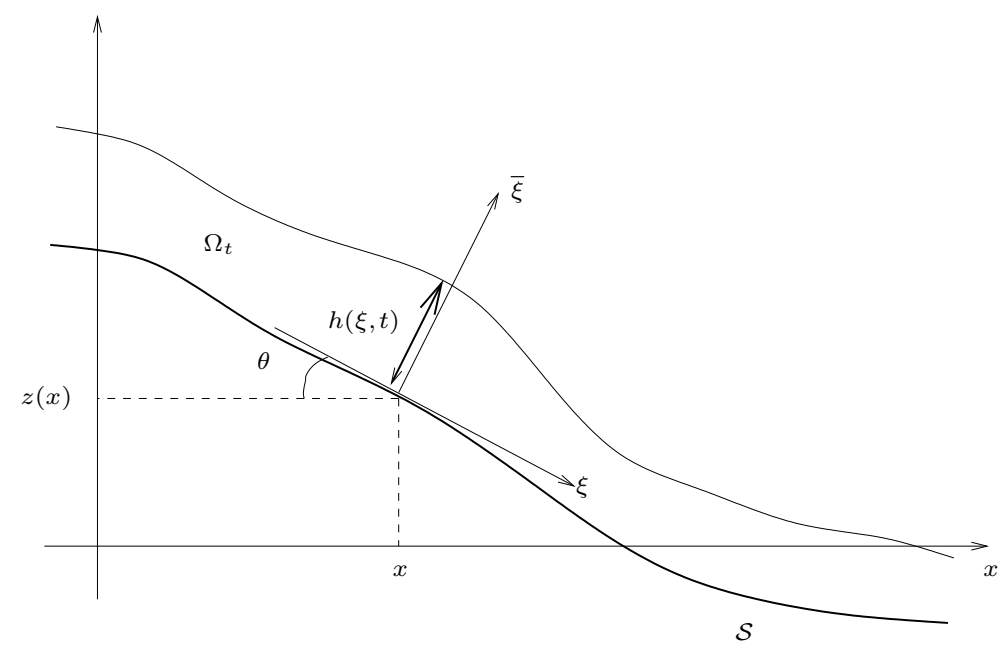

FIG. 2.1. Curvilinear coordinates

equations.

\section{Navier-Stokes equations with free surface in curvilinear coordinates}

In this section, we write the Navier-Stokes equations in a system of coordinates adapted to the geometry of the fluid layer. We first describe the change of variables from cartesian coordinates to a system of coordinates adapted to the bottom surface geometry and show how the principal differential operators are transformed. We decompose the fluid speed in this new reference frame and reformulate both the boundary conditions and the Navier-Stokes equations with these new coordinates and variables.

2.1. Curvilinear coordinates. Let $y=z(x), x \in \mathbb{R}^{n}, n=1,2$ be the graph of the function describing the bottom surface $\mathcal{S}$. The normal $\mathbf{n}$ to $\mathcal{S}$ is given by

$$
\mathbf{n}=\left(1+\left\|\nabla_{x} z\right\|^{2}\right)^{-\frac{1}{2}}\left(\begin{array}{c}
-\nabla_{x} z \\
1
\end{array}\right)=\left(\begin{array}{c}
-\mathbf{s} \\
c
\end{array}\right),
$$

where $\|$.$\| is the classical euclidian norm in \mathbb{R}^{n}$. The scalar $c$ is the cosine of the angle $\theta$ between $\mathbf{n}$ and the vertical (see Fig. 2.1). From this definition and $c^{2}+\|\mathbf{s}\|^{2}=1$, we notice that

$$
\partial_{x} c=-\frac{1}{c} s^{t} \partial_{x} \mathbf{s}, \partial_{x} \mathbf{s}=c\left(1-\mathbf{s s}^{t}\right) \partial_{x x}^{2} z, \partial_{x x}^{2} z=\frac{c^{2} I d+\mathbf{s s}^{t}}{c^{3}} \partial_{x} \mathbf{s} .
$$

Here, $\mathcal{H}_{b}=c \partial_{x x}^{2} z$ denotes the curvature tensor of the bottom surface and $\partial_{x} \mathbf{s}=(1-$ $\left.\mathbf{s s}^{t}\right) \mathcal{H}_{b}$. It is sometimes convenient not to work in cartesian coordinates, but in a coordinate system adapted to the topography. This is the point of view adopted here. In view of the fact that the models must be solved numerically, it is important to have some flexibility in the choice of coordinates in which our models are written. In what follows, we assume that a parametrization of the bottom surface $\xi \in \mathbb{R}^{n} \mapsto x(\xi) \in \mathbb{R}^{n}$ is given. 
Define $\vec{\xi}=(\xi, \bar{\xi})$, a new system of coordinates, where $\xi \in \mathbb{R}^{n}$ is a curvilinear abscissa along the bottom and $\bar{\xi} \in \mathbb{R}$ the signed distance in the direction of the normal. The horizontal coordinate $x$ is given by the parametrization $\xi \mapsto x(\xi)$. Denote by $\partial_{\xi} x$ the Jacobian matrix of the transformation. We assume for convenience that $\operatorname{det}\left(\partial_{\xi} x\right)>0$. In what follows, the fluid domain $\Omega_{t}$ is defined as

$$
\vec{X} \in \Omega_{t} \Leftrightarrow \vec{X}(\xi, \bar{\xi})=\left(\begin{array}{c}
x(\xi) \\
z(x(\xi))
\end{array}\right)+\bar{\xi}\left(\begin{array}{c}
-\mathbf{s}(x(\xi)) \\
c(x(\xi))
\end{array}\right), \quad 0 \leq \bar{\xi} \leq h(\xi, t),
$$

where $h$ is the fluid height. In the following, we shall use the same notations introduced in $[2]$.

It is natural to define new velocity components using a decomposition of the velocity $\vec{U}$ by Jacobian matrix:

$$
\vec{U}=\left(\partial_{\vec{\xi}} \vec{X}\right) \vec{V} \Longleftrightarrow\left\{\begin{array}{l}
U=\left(\partial_{\xi} X\right) V-\bar{V} \mathbf{s} \\
\bar{U}=\frac{1}{c} \mathbf{s}^{t}\left(\partial_{\xi} X\right) V+c \bar{V}
\end{array}\right.
$$

with $\partial_{\xi} X=\left(\operatorname{Id}-\bar{\xi} \partial_{x} \mathbf{s}\right) \partial_{\xi} x, U, V \in \mathbb{R}^{n}, \bar{U}, \bar{V} \in \mathbb{R}$. There are other possible choices of decomposition: see [2] for further details.

Let us define the matrix $A \in M_{n+1}(\mathbb{R})$ as

$$
A=\left(\partial_{\vec{\xi}} \vec{X}\right)^{-1}=\left(\begin{array}{cc}
\left(\partial_{\xi} X\right)^{-1} & 0 \\
0 & 1
\end{array}\right)\left(\begin{array}{cc}
\mathrm{Id}-\mathbf{s s}^{t} & c \mathbf{s} \\
-\mathbf{s}^{t} & c
\end{array}\right) .
$$

For further reference, we compute $\widetilde{M}=A A^{t} \in M_{n+1}(\mathbb{R})$ :

$$
\widetilde{M}=\left(\begin{array}{rr}
M & 0 \\
0 & 1
\end{array}\right), M=\left(\partial_{\xi} X\right)^{-1}\left(\operatorname{Id}-\mathbf{s s}^{t}\right)\left(\partial_{\xi} X\right)^{-t} \in M_{n}(\mathbb{R}) .
$$

The Jacobian $J$ of the transformation is given by

$$
J=\operatorname{det}\left(\partial_{\vec{\xi}} \vec{X}\right)=\frac{1}{c} \operatorname{det}\left(\partial_{\xi} X\right)=\operatorname{det}(M)^{-\frac{1}{2}} .
$$

Let us describe how the principal differential operators used in the Navier-Stokes equations are transformed through this change of variables.

Lemma 2.1. For any vector field $\vec{Z}$ and any symmetric tensor $\sigma=\sigma^{t}$, the differential operators transform according to

$$
\begin{aligned}
& J \nabla_{\vec{X}} \cdot \vec{Z}=\nabla_{\vec{\xi}} \cdot(J A \vec{Z}), \quad \vec{U} \cdot \nabla_{\vec{X}}=\vec{V} \cdot \nabla_{\vec{\xi}}, \quad \nabla_{\vec{X}}=A^{t} \nabla_{\vec{\xi}}, \\
& J A^{-t} \nabla_{\vec{X}} \cdot \sigma=\nabla_{\vec{\xi}} \cdot\left(J \mathcal{P} A A^{t}\right)+\frac{J}{2} \mathcal{P}: \nabla_{\vec{\xi}}\left(A A^{t}\right),
\end{aligned}
$$

with $\mathcal{P}=A^{-t} \sigma A^{-1}$.

For the proof of this lemma, see [2].

2.2. Reformulation of the Navier-Stokes equations. The Navier-Stokes equations for the fluid velocity $\vec{U}$ and the pressure $p$ in the domain $\Omega_{t}$ read

$$
\begin{aligned}
& \partial_{t} \vec{U}+\vec{U} \cdot \nabla_{\vec{X}} \vec{U}+\nabla_{\vec{X}} p=-\vec{g}+\mu \nabla_{\vec{X}} \cdot \sigma, \\
& \nabla_{\vec{X}} \cdot \vec{U}=0 .
\end{aligned}
$$


Here $\sigma=\partial_{\vec{X}} \vec{U}+\left(\partial_{\vec{X}} \vec{U}\right)^{t}$ is the deformation tensor, $\mu$ measures the fluid viscosity and the vector $\vec{g}$ represents the gravity forces. The density of the fluid is supposed to be constant and set to 1 . These equations come with boundary conditions. We aim to write the Navier-Stokes equations (2.8) and boundary conditions in the reference frame introduced in the previous section with the Jacobian decomposition of the velocity.

2.2.1. Boundary conditions. The Navier-Stokes equations (2.8) come with boundary conditions at the bottom $\bar{\xi}=0$ and at the free surface $\bar{\xi}=h(\xi, t)$. More precisely, we assume a no-slip condition at the bottom

$$
V(\xi, 0)=0, \quad \bar{V}(\xi, 0)=0, \quad \forall \xi \in \mathbb{R}^{n} .
$$

At the free surface $\bar{\xi}=h(\xi, t)$, we write that the fluid layer $\Omega_{t}$ is advected by the fluid speed $\vec{V}$ : this yields the mass conservation condition (or equivalently an impermeability condition):

$$
h_{t}+V(., h) . \nabla_{\xi} h=\bar{V}(., h) .
$$

The other boundary condition at the free surface $\bar{\xi}=h(\xi, t)$ is the continuity of fluid stress at that interface. For completeness, we take into account the capillary effects. The atmospheric pressure $p_{\text {atm }}$ is constant and set to zero. Then the continuity of fluid stress reads

$$
(\sigma-p \mathrm{Id}) \vec{N}=\kappa \mathcal{H} \vec{N}, \quad \forall \bar{\xi}=h(\xi, t) .
$$

The scalar $\mathcal{H}$ is the mean curvature of the free surface, $\kappa$ measures the capillary effects and $\vec{N}$ is the normal to the free surface. We are going to describe more precisely the continuity of fluid stress with the help of the new variables and the Jacobian speed $\vec{V}$. Let us first compute $\vec{N}$.

Lemma 2.2. The unitary normal to the free surface $\bar{\xi}=h(\xi, t)$ is given by

$$
\vec{N}=\alpha A^{t}\left(\begin{array}{c}
-\nabla_{\xi} h \\
1
\end{array}\right)
$$

where $\alpha^{-1}=\left\|A^{t}\left(\begin{array}{c}-\nabla_{\xi} h \\ 1\end{array}\right)\right\|$.

The proof of this lemma is straightforward and can be found in [2]. Now associated to the deformation tensor $\sigma$, introduce $\mathcal{P}$ as

$$
\mathcal{P}=A^{-t} \sigma A^{-1}=\left(\begin{array}{cc}
P & Z \\
Z^{t} & f
\end{array}\right) .
$$

Lemma 2.3. The terms $P, Z, f$ of the tensor $\mathcal{P}=A^{-t} \sigma A^{-1}$ with

$$
\sigma=\partial_{\vec{X}} \vec{U}+\left(\partial_{\vec{X}} \vec{U}\right)^{t}
$$

are given by

$$
\begin{aligned}
& Z=\partial_{\bar{\xi}}\left(M^{-1} V\right)+\nabla_{\xi} \bar{V}+2 \partial_{x} \mathbf{s}^{t}\left(\operatorname{Id}+\frac{\mathbf{s s}^{t}}{c^{2}}\right)\left(\partial_{\xi} X\right) V, \\
& f=2 \partial_{\bar{\xi}} \bar{V}, P=Q+Q^{t}
\end{aligned}
$$


where

$$
\begin{aligned}
Q= & \partial_{\xi}\left(M^{-1} V\right)+\partial_{\xi}\left(\left(\partial_{\xi} X\right)^{t}\right) \cdot\left(\left(\partial_{\xi} X\right) V-\bar{V} \mathbf{s}\right) \\
& +\left(\frac{\mathbf{s}^{t}}{c}\left(\partial_{\xi} X\right) V+c \bar{V}\right) \partial_{\xi}\left(\frac{\left(\partial_{\xi} X\right)^{t}}{c} \mathbf{s}\right) .
\end{aligned}
$$

In the new system of coordinates, the continuity of the fluid stress (2.11) at the free surface $\bar{\xi}=h(\xi, t)$ can be written

$$
\mathcal{P}_{h} \widetilde{M}_{h}\left(\begin{array}{c}
-\nabla_{\xi} h \\
1
\end{array}\right)-p_{h}\left(\begin{array}{c}
-\nabla_{\xi} h \\
1
\end{array}\right)=\kappa \mathcal{H}\left(\begin{array}{c}
-\nabla_{\xi} h \\
1
\end{array}\right),
$$

where the subscript $p_{h}\left(\right.$ resp. $\left.\mathcal{P}_{h}, \widetilde{M}_{h}\right)$ is the value of $p($ resp. $\mathcal{P}, \widetilde{M})$ at the free surface. In the following, we shall note the value of flow unknowns at the free surface with a similar subscript. Finally we can write the system (2.14) in the form

$$
\begin{aligned}
& \mu P_{h} M_{h} \nabla_{\xi} h+\mu Z_{h}+p_{h} M_{h} \nabla_{\xi} h=-\kappa \mathcal{H} \nabla_{\xi} h, \\
& -\mu\left(M_{h} Z_{h}\right)^{t} \nabla_{\xi} h+\mu f_{h}-p_{h}=\kappa \mathcal{H} .
\end{aligned}
$$

2.2.2. Free divergence equation and momentum equation. In this section, we write the Navier-Stokes equations using the curvilinear coordinates and the Jacobian decomposition of the velocity. Using chain rules (2.7), the divergence-free condition yields

$$
J \nabla_{\vec{X}} \cdot \vec{U}=\nabla_{\vec{\xi}} \cdot(J \vec{V})=\nabla_{\xi} \cdot(J V)+\partial_{\bar{\xi}} \bar{V}=0 .
$$

Multiplying the momentum equation in (2.8) by the matrix $A$, one finds

$$
\begin{aligned}
\partial_{t} \vec{V}+A\left(\vec{V} \cdot \nabla_{\vec{\xi}}\right)\left(A^{-1} \vec{V}\right) & +\widetilde{M} \nabla_{\vec{\xi}} p=-A \vec{g} \\
& +\frac{\mu}{J} \widetilde{M}\left(\nabla_{\vec{\xi}} \cdot(J \mathcal{P} \widetilde{M})+\frac{J}{2} \mathcal{P}: \nabla_{\vec{\xi}} \widetilde{M}\right) .
\end{aligned}
$$

We decompose the advection term $A\left(\vec{V} \cdot \nabla_{\vec{\xi}}\right)\left(A^{-1} \vec{V}\right)$ in the form

$$
A\left(\vec{V} \cdot \nabla_{\vec{\xi}}\right)\left(A^{-1} \vec{V}\right)=\vec{V} \cdot \nabla_{\vec{\xi}} \vec{V}-\vec{\Gamma}(\vec{V}),
$$

with

$$
\vec{\Gamma}(\vec{V})=\left(\begin{array}{c}
\Gamma(\vec{V}) \\
\bar{\Gamma}(\vec{V})
\end{array}\right)=\left(\begin{array}{c}
\left(\partial_{\xi} X\right)^{-1}\left(-\left(\partial_{\xi \xi}^{2} X\right) \cdot V \cdot V+2 \bar{V}\left(\partial_{\xi} \mathbf{s}\right) V+\bar{\Gamma}(\vec{V}) \mathbf{s}\right) \\
-c V^{t}\left(\partial_{\xi} x\right)^{t}\left(\partial_{x x}^{2} z\right)\left(\partial_{\xi} X\right) V
\end{array}\right)
$$

The momentum equation (2.17) can be written

$$
\begin{aligned}
\partial_{t} \vec{V}+\vec{V} \cdot \nabla_{\vec{\xi}} \vec{V}+\widetilde{M} \nabla_{\vec{\xi}} p= & -A \vec{g}+\vec{\Gamma}(\vec{V}) \\
& +\frac{\mu}{J} \widetilde{M}\left(\nabla_{\vec{\xi}} \cdot(J \mathcal{P} \widetilde{M})+\frac{J}{2} \mathcal{P}: \nabla_{\vec{\xi}} \widetilde{M}\right) .
\end{aligned}
$$

Note that the vector $\vec{\Gamma}(\vec{V})$ has a physical interpretation: it represents the centrifugal forces experienced by the fluid and associated to the change of reference frame.

We aim to write a shallow water model for the fluid layer: it consists essentially of an equation of mass conservation and an equation for the streamwise velocity averaged 
along the fluid height. For this purpose, we separate the equation for the horizontal velocity $V$ and vertical velocity $\bar{V}$, and obtain the system

$$
\begin{aligned}
\partial_{t} V+\vec{V} \cdot \nabla_{\vec{\xi}} V & +M \nabla_{\xi} p=-g c\left(\partial_{\xi} X\right)^{-1} \mathbf{s}+\Gamma(\vec{V}) \\
& +\frac{\mu}{J} M\left(\nabla_{\xi} \cdot(J P M)+\partial_{\bar{\xi}}(J Z)+\frac{J}{2} P: \nabla_{\xi} M\right), \\
\partial_{t} \bar{V}+\vec{V} \cdot \nabla_{\vec{\xi}} \bar{V}+ & \partial_{\bar{\xi}} p=-g c+\bar{\Gamma}(\vec{V}) \\
& +\frac{\mu}{J}\left(\nabla_{\xi} \cdot(J M Z)+\partial_{\bar{\xi}}(J f)+\frac{J}{2} P: \partial_{\bar{\xi}} M\right) .
\end{aligned}
$$

2.3. Averaged equations. In what follows, we obtain exact evolution equations for the fluid height $h$ and the streamwise velocity averaged along the fluid height. This system is composed of an equation of mass conservation and a momentum equation. Let us first write the equation of mass conservation with average quantities. In the following, all the integrals computed are integrals of functions with respect to the cross-stream variable $\bar{\xi}$ : for a function $f(\xi, \bar{\xi})$, we shall write

$$
\int f=\int f(\xi, \bar{\xi}) d \bar{\xi}
$$

and the bounds of integration are specified for each calculation.

Integrating the divergence free condition $(2.16)$ over $(0, h)$ yields

$$
J_{h} \bar{V}_{h}+\int_{0}^{h} \nabla_{\xi} \cdot(J V)=J_{h} \bar{V}_{h}+\nabla_{\xi} \cdot\left(\int_{0}^{h} J V\right)-J_{h} V_{h} \cdot \nabla_{\xi} h=0 .
$$

Here and in the following, the subscripted quantity $J_{h}$ (resp. $V_{h}, \bar{V}_{h}, p_{h}$, etc.) denotes the value of $J$ (resp. $V, \bar{V}, p$ ) at the free surface $\bar{\xi}=h(\xi, t)$. Using the mass conservation condition (2.10), one finds

$$
J_{h} \partial_{t} h+\nabla_{\xi} \cdot\left(\int_{0}^{h} J V\right)=0
$$

Since $J$ is time-independent, Equation (2.24) can also be written

$$
\partial_{t}\left(\int_{0}^{h} J\right)+\nabla_{\xi} \cdot\left(\int_{0}^{h} J V\right)=0
$$

In order to write the mass conservation equation in a simple form, we introduce the average quantities

$$
\tilde{h}=\int_{0}^{h} J, \quad \tilde{h} \tilde{v}=\int_{0}^{h} J V
$$

and the mass conservation equation $(2.25)$ reads

$$
\tilde{h}_{t}+\nabla_{\xi} \cdot(\tilde{h} \tilde{v})=0 .
$$

In the following, we shall write an evolution system for the more natural quantities $(\tilde{h}, \tilde{h} \tilde{v})$. We will see later that under suitable hypothesis, we can deduce $h$ and $\int_{0}^{h} V$ 
from $\tilde{h}$ and $\tilde{h} \tilde{v}$. Integrating the left hand side of Equation (2.20) over the interval $(0, h)$ yields

$$
\begin{aligned}
\int_{0}^{h} J\left(\partial_{t} V+\vec{V} \cdot \nabla_{\vec{\xi}} V+M \nabla_{\xi} p\right)= & \partial_{t}\left(\int_{0}^{h} J V\right)+\nabla_{\xi} \cdot\left(\int_{0}^{h} J V \otimes V\right) \\
& +\nabla_{\xi} \cdot\left(\int_{0}^{h} J M p\right)-\int_{0}^{h} p \nabla_{\xi} \cdot(J M) \\
& +J_{h}\left(h_{t}+V_{h} \cdot \nabla_{\xi} h-\bar{V}_{h}\right) V_{h}-J_{h} p_{h} M_{h} \nabla_{\xi} h .
\end{aligned}
$$

Furthermore, we integrate the right hand side of (2.20) and use the boundary conditions (2.15). We find

$$
\begin{aligned}
& \partial_{t}\left(\int_{0}^{h} J V\right)+\nabla_{\xi} \cdot\left(\int_{0}^{h} J V \otimes V+J p M\right) \\
= & -g c \int_{0}^{h} J\left(\partial_{\xi} X\right)^{-1} \mathbf{s}-\mu J_{0} M_{0} Z_{0}+\int_{0}^{h} J \Gamma(\vec{V})+\int_{0}^{h} p \nabla_{\xi} \cdot(J M) \\
& +\frac{\mu}{2} \int_{0}^{h} J M\left(P: \nabla_{\xi} M\right)-\mu \int_{0}^{h} J\left(\partial_{\bar{\xi}} M\right) Z \\
& +\mu \nabla_{\xi} \cdot \int_{0}^{h} J M P M-\mu \int_{0}^{h} J P M:: \partial_{\xi} M \\
& +\mu J_{h} M_{h} Z_{h}-\mu J_{h} M_{h} P_{h} M_{h} \nabla_{\xi} h+J_{h} p_{h} M_{h} \nabla_{\xi} h,
\end{aligned}
$$

where $\left(A:: \partial_{\xi} B\right)$ is the operator defined by $\left(A:: \partial_{\xi} B\right)_{i}=\operatorname{tr}\left(A \partial_{\xi} B^{i}\right), B^{i}$ being the $i$-th column of $B$. The continuity of the fluid stress at the free surface yields

$$
\mu J_{h} M_{h} Z_{h}-\mu J_{h} M_{h} P_{h} M_{h} \nabla_{\xi} h+J_{h} p_{h} M_{h} \nabla_{\xi} h=-\kappa \mathcal{H} J_{h} M_{h} \nabla_{\xi} h .
$$

As a consequence, the equation for the average momentum $\int_{0}^{h} J V=\tilde{h} \tilde{v}$ reads

$$
\begin{aligned}
& \partial_{t}\left(\int_{0}^{h} J V\right)+\nabla_{\xi} \cdot\left(\int_{0}^{h} J V \otimes V+J p M\right)+\kappa \mathcal{H} J_{h} M_{h} \nabla_{\xi} h \\
= & -g c \int_{0}^{h} J\left(\partial_{\xi} X\right)^{-1} \mathbf{s}-\mu J_{0} M_{0} Z_{0}+\int_{0}^{h} J \Gamma(\vec{V}) \\
& +\int_{0}^{h} p \nabla_{\xi} \cdot(J M)+\frac{\mu}{2} \int_{0}^{h} J M\left(P: \nabla_{\xi} M\right)-\mu \int_{0}^{h} J\left(\partial_{\bar{\xi}} M\right) Z \\
& +\mu \nabla_{\xi} \cdot \int_{0}^{h} J M P M-\mu \int_{0}^{h} J P M:: \partial_{\xi} M .
\end{aligned}
$$

This equation is exact and no approximation has been done for the moment in order to obtain an evolution system for $(\tilde{h}, \tilde{h} \tilde{v})$ in a closed form: this shall be done in the forthcoming section.

\section{Shallow water asymptotics}

In the following, we derive the shallow water equations from the Navier-Stokes system $(2.16,2.17)$ and boundary conditions $(2.9,2.10,2.15)$; scaling these equations, we shall specify in which parameter regime the classical assumptions made for the 
derivation of the shallow water equations (hydrostatic pressure, quasi-stationary flow) hold true. Then we compute an asymptotic expansion of the Navier-Stokes equations with respect to the aspect ratio, $\varepsilon=\frac{H}{L} \ll 1$, between the characteristic height of the fluid, and $L$, the characteristic wavelength of the solutions; inserting these approximations into the averaged equation (2.29) and dropping "small" terms, we deduce an evolution system for $\widetilde{h}$ and $\widetilde{h} \widetilde{v}=\int_{0}^{h} J V$ in a closed form.

3.1. Scaling of the equations. Let $U_{0}$ and $H$ be respectively the characteristic velocity and height of a flow. We define classically some dimensionless numbers, respectively the Froude number $\mathbf{F}$, the Reynolds number $\mathbf{R e}$ and the Weber number We:

$$
\mathbf{F}=\frac{U_{0}}{\sqrt{g H}}, \quad \mathbf{R e}=\frac{H U_{0}}{\mu}, \quad \mathbf{W e}=\frac{H U_{0}^{2}}{\kappa} .
$$

We do not make precise here the characteristic speed $U_{0}$; nevertheless, several choices are possible. As an example, for an inclined plane with a non-zero slope $\phi$, the stationary Nusselt solution is given by

$$
h=H, \quad \bar{V}=0, \quad V=\frac{g \sin (\phi)}{\mu}\left(H \bar{\xi}-\frac{\bar{\xi}^{2}}{2}\right) .
$$

Thus, in this case a typical choice of speed $U_{0}$ could be $U_{0}=\frac{g H^{2}}{2 \mu} \sin (\phi)$, and $\mathbf{F}^{2}=\frac{\mathbf{R e}}{2}$.

In the following, we consider functions with a characteristic wavelength $L$ such that the aspect ratio $\varepsilon=H / L \ll 1$. We introduce the scaling

$$
\begin{aligned}
& x=L \widetilde{x}, \quad \xi=L \widetilde{\xi}, \quad h=H \widetilde{h}, \quad \bar{\xi}=H \widetilde{\bar{\xi}}, \quad t=\frac{L}{U_{0}} \widetilde{t} \\
& V=U_{0} \widetilde{V}, \quad \bar{V}=\frac{H}{L} U_{0} \widetilde{\bar{V}}, \quad p=g H \widetilde{p} .
\end{aligned}
$$

Let us describe the bottom surface in this scaling. In order to deal with arbitrary topography, we will suppose that $\mathbf{s}=\mathcal{O}(1)$. This means that $\nabla_{x} z=\mathcal{O}(1)$ and imposes the scaling on $z=L \widetilde{z}$. Let us describe the curvature of the bottom surface. Here, we want to separate the long wavelength assumption on the free surface and the curvature of the bottom surface. For that purpose, we assume that the curvature of the surface is of order $\mathcal{O}(1 / R)$ where $R$ is the characteristic radius of curvature of the bottom surface and is not necessarily of order $\mathcal{O}(L)$. Then, the derivatives $\partial_{x} \mathbf{s}$ and $\partial_{x x}^{2} z$ are $\mathcal{O}(1 / R)$. Let us introduce $\theta_{R}=\frac{L}{R}$, the ratio between the characteristic wavelength of flow variables, and $R$, the characteristic curvature of the bottom surface, and define the rescaled bottom curvature $\mathcal{H}_{b}=\frac{\theta_{R}}{L} \widetilde{\mathcal{H}}_{b}$. We shall see later that choosing different values for $\theta_{R}$ yields different type of models, in particular the influence of capillarity.

Here we have introduced the parameter $\theta_{R}$ coherent with the scaling, which describes the typical curvature of the bottom surface. It is important to note that we have made no restriction on the amplitude of the inclination and curvature of the bottom: the size of these parameters with respect to $\varepsilon$, especially the characteristic curvature $\theta_{R}$, will be discussed at the end of the paper to distinguish different models.

For the other quantities $\Gamma, \mathcal{H}, P, Z, f$, we choose the natural scaling:

$$
\Gamma=\frac{U^{2}}{L} \widetilde{\Gamma}, \quad \mathcal{H}=\frac{1}{L} \widetilde{\mathcal{H}}, \quad P=\frac{U}{L} \widetilde{P}, \quad f=\frac{U}{L} \widetilde{f}, \quad Z=\frac{U}{H} \widetilde{Z} .
$$


Now we write the Navier-Stokes equations in a non-dimensional form. Dropping the $\sim$ from the equations $(2.20),(2.21)$, we find:

$$
\begin{aligned}
\partial_{t} V+\vec{V} \cdot \nabla_{\vec{\xi}} V+\frac{M}{\mathbf{F}^{2}} \nabla_{\xi} p= & -\frac{1}{\varepsilon \mathbf{F}^{2}} c\left(\partial_{\xi} X\right)^{-1} \mathbf{s} \\
& +\left(\partial_{\xi} X\right)^{-1}\left(\theta_{R} \bar{\Gamma}(\vec{V}) \mathbf{s}-\left(\partial_{\xi \xi}^{2} X\right) \cdot V \cdot V+2 \varepsilon \theta_{R} \bar{V}\left(\partial_{\xi} \mathbf{s}\right) V\right) \\
& +\frac{1}{J \varepsilon \mathbf{R e}}\left(\partial_{\bar{\xi}}(J \bar{Z})+\varepsilon^{2}\left(\nabla_{\xi} \cdot(J \bar{P} M)+\frac{J}{2} \bar{P}: \nabla_{\xi} M\right)\right) \\
\partial_{t} \bar{V}+\vec{V} \cdot \nabla_{\vec{\xi}} \bar{V}+\frac{1}{\varepsilon^{2} \mathbf{F}^{2}} \partial_{\bar{\xi}} p= & -\frac{1}{\varepsilon^{2} \mathbf{F}^{2}} c+\frac{\theta_{R}}{\varepsilon} \bar{\Gamma}(\vec{V}) \\
& +\frac{1}{J \varepsilon \mathbf{R e}}\left(\nabla_{\xi} \cdot(J M \bar{Z})+\partial_{\bar{\xi}}(J \bar{f})+\frac{J}{2} \bar{P}: \partial_{\bar{\xi}} M\right) .
\end{aligned}
$$

Note that, in these equations, the rescaled quantities related to the change of reference frame are given by

$$
\begin{aligned}
& J=\frac{\operatorname{det}\left(\partial_{\xi} x\right)}{c} \operatorname{det}\left(\operatorname{Id}-\varepsilon \theta_{R} \bar{\xi}\left(\partial_{x} \mathbf{s}\right)\right), \quad \partial_{\xi} X=\left(\operatorname{Id}-\varepsilon \theta_{R} \bar{\xi}\left(\partial_{x} \mathbf{s}\right)\right) \partial_{\xi} x \\
& M=\left(\partial_{\xi} x\right)^{-1}\left(\operatorname{Id}-\varepsilon \theta_{R} \bar{\xi}\left(\partial_{x} \mathbf{s}\right)\right)\left(\operatorname{Id}-\mathbf{s} \mathbf{s}^{t}\right)\left(\operatorname{Id}-\varepsilon \theta_{R} \bar{\xi}\left(\partial_{x} \mathbf{s}\right)^{t}\right)\left(\partial_{\xi} x\right)^{-t}
\end{aligned}
$$

whereas the rescaled quantites associated to the flow variables read:

$$
\begin{aligned}
& \bar{Z}=\partial_{\xi}\left(M^{-1} V\right)+2 \varepsilon \theta_{R}\left(\partial_{\xi} \mathbf{s}\right)^{t}\left(\mathrm{Id}+\frac{\mathbf{s s}^{t}}{c}\right) \partial_{\xi} X V+\varepsilon^{2} \nabla_{\xi} \bar{V}, \\
& \bar{f}=2 \partial_{\bar{\xi}} \bar{V}, \quad \bar{P}=\bar{Q}+\bar{Q}^{t}
\end{aligned}
$$

with $\bar{Q}$ defined as

$$
\begin{aligned}
\bar{Q}= & \partial_{\xi}\left(M^{-1} V\right)+\partial_{\xi}\left(\left(\partial_{\xi} X\right)^{t}\right) \cdot\left(\left(\partial_{\xi} X\right) V-\varepsilon \bar{V} \mathbf{s}\right) \\
& +\left(\frac{\mathbf{s}^{t}}{c}\left(\partial_{\xi} X\right) V+\varepsilon c \bar{V}\right) \frac{\left(\partial_{\xi} X\right)^{t} \mathbf{s}}{c}
\end{aligned}
$$

The particular choice of characteristic velocity for the vertical velocity $\bar{V}$ implies that the divergence-free condition (2.16) is preserved under the shallow water scaling

$$
\nabla_{\xi} \cdot V+\partial_{\bar{\xi}} \bar{V}=0
$$

We write the rescaled boundary conditions at the bottom $\bar{\xi}=0$ and at the free surface $\bar{\xi}=h(\xi, t)$. On the one hand, the no-slip conditions at the bottom and the impermeability condition (see (2.9) and (2.10)) are unchanged under the shallow water scaling:

$$
\begin{aligned}
& V(., \bar{\xi}=0)=0, \bar{V}(., \bar{\xi}=0)=0, \\
& \partial_{t} h+V_{h} . \nabla_{\xi} h=\bar{V}_{h} .
\end{aligned}
$$

On the other hand, the continuity of fluid stress (2.15) at the free surface reads

$$
\begin{aligned}
& \frac{\varepsilon}{\mathbf{F}^{2}} p_{h} M_{h} \nabla_{\xi} h+\frac{1}{\mathbf{R e}} M_{h} \bar{Z}_{h}-\frac{\varepsilon^{2}}{\mathbf{R e}} M_{h} \bar{P}_{h} M_{h} \nabla_{\xi} h=-\frac{\varepsilon^{2} \mathcal{H}}{\mathbf{W e}} M_{h} \nabla_{\xi} h, \\
& \frac{\varepsilon}{\mathbf{R e}}\left(\bar{f}_{h}-\left(M_{h} \bar{Z}_{h}\right)^{t} \nabla_{\xi} h\right)-\frac{1}{\mathbf{F}^{2}} p_{h}=\frac{\varepsilon \mathcal{H}}{\mathbf{W e}} .
\end{aligned}
$$


Finally, we write the nondimensional counterpart of the averaged equations (2.25) and (2.29); this yields exact evolution equation for

$$
\widetilde{h}=\int_{0}^{h} J, \quad \widetilde{h} \widetilde{v}=\int_{0}^{h} J V
$$

where $h, J, V$ are the rescaled flow variables. The dimensionless continuity equation reads

$$
\partial_{t}\left(\int_{0}^{h} J\right)+\nabla_{\xi} \cdot\left(\int_{0}^{h} J V\right)=0
$$

Integrating Equation (3.3) over the interval $(0, h)$ and using the rescaled boundary conditions yields the averaged momentum equation:

$$
\begin{aligned}
& \partial_{t}\left(\int_{0}^{h} J V\right)+\nabla_{\xi} \cdot\left(\int_{0}^{h} J V \otimes V\right)+\frac{1}{\mathbf{F}^{2}} \nabla_{\xi} \cdot\left(\int_{0}^{h} J p M\right)+\frac{\varepsilon \mathcal{H}}{\mathbf{W e}} J_{h} M_{h} \nabla_{\xi} h \\
= & -\frac{1}{\varepsilon \mathbf{F}^{2}} c \int_{0}^{h} J\left(\partial_{\xi} X\right)^{-1} \mathbf{s}-\frac{1}{\varepsilon \mathbf{R e}} J_{0} \partial_{\bar{\xi}} V_{0} \\
& +\int_{0}^{h} J\left(\partial_{\xi} X\right)^{-1}\left(\theta_{R} \bar{\Gamma}(\vec{V}) \mathbf{s}-\left(\partial_{\xi \xi}^{2} X\right) \cdot V \cdot V+2 \varepsilon \theta_{R} \bar{V}\left(\partial_{x} \mathbf{s}\right) V\right) \\
& +\frac{1}{\mathbf{F}^{2}} \int_{0}^{h} p \nabla_{\xi} \cdot(J M)-\frac{1}{\varepsilon \mathbf{R e}} \int_{0}^{h} J\left(\partial_{\bar{\xi}} M\right) \bar{Z} \\
& +\frac{\varepsilon}{\mathbf{R e}}\left(\nabla_{\xi} \cdot \int_{0}^{h} J M \bar{P} M+\int_{0}^{h} \frac{J}{2} M\left(\bar{P}: \nabla_{\xi} M\right)-J \bar{P} M:: \partial_{\xi} M\right) .
\end{aligned}
$$

3.2. Asymptotics. In the following, we write an evolution system for $\tilde{h}=\int_{0}^{h} J$ and $\tilde{h} \tilde{v}=\int_{0}^{h} J V$. Recall the continuity equation (3.12)

$$
\partial_{t} \widetilde{h}+\nabla_{\xi} \cdot(\widetilde{h} \widetilde{v})=0
$$

We shall write an approximate evolution equation for $\tilde{h} \tilde{v}$ using (3.13). For that purpose, we assume that the aspect ratio $\varepsilon=\frac{H}{L} \ll 1$ and we introduce the dimensionless parameters

$$
\alpha=\frac{\varepsilon \mathbf{F}^{2}}{\mathbf{R e}}, \quad \beta=\varepsilon \mathbf{R e}, \quad \delta=\frac{\varepsilon \mathbf{R e}}{\mathbf{F}^{2}}, \quad \lambda=\frac{\mathbf{R e}}{\mathbf{F}^{2}}, \quad \bar{\kappa}=\frac{\varepsilon \mathbf{F}^{2}}{\mathbf{W e}} .
$$

From the assumption $\varepsilon \ll 1$ and considering the Reynolds number $\mathbf{R e}$ and the Froude number $\mathbf{F}$ to be of order one, we deduce that $\alpha, \beta, \delta \ll 1$ and $\lambda=\mathcal{O}(1)$. In fact, we shall see later that the expansions carried out in what follows remain valid provided that $\alpha, \beta, \delta \ll 1$; thus, the range of validity of the models obtained here is not restricted to Reynolds and Froude numbers of order one. In particular, we can choose $\mathbf{R e} \rightarrow \infty$ as $\varepsilon \rightarrow 0$. To see the influence of the capillary effects, we shall assume that $\bar{\kappa}=\mathcal{O}(1)$.

We now study the influence of the shallow water scaling on the Navier-Stokes equations. Using Equation (3.4), a straightforward computation yields an equation 
for the pressure:

$$
\begin{aligned}
\partial_{\bar{\xi}} p+c= & \frac{\alpha}{J}\left(\nabla_{\xi} \cdot(J M \bar{Z})+\partial_{\bar{\xi}}(J \bar{f})+\frac{J}{2} \bar{P}: \partial_{\bar{\xi}} M\right) \\
& +\frac{\theta_{R} \beta}{\lambda} \bar{\Gamma}(\vec{V})-\alpha \beta\left(\partial_{t} \bar{V}+\vec{V} \cdot \nabla_{\vec{\xi}} \bar{V}\right)=: \Psi_{p}(\vec{V}, \vec{\xi}, t) .
\end{aligned}
$$

In the asymptotic regime $\alpha, \beta \ll 1, \theta_{R}, \lambda=\mathcal{O}(1)$, we clearly recover the classical assumption of hydrostatic pressure made in the derivation of shallow water equations. Moreover, the continuity of the fluid stress at the free boundary $\bar{\xi}=h$ implies that the pressure at the free surface is given by (see Equation (3.9)):

$$
p_{h}=-\bar{\kappa} \mathcal{H}+\alpha\left(\bar{f}_{h}-\left(M_{h} \bar{Z}_{h}\right)^{t} \nabla_{\xi} h\right):=\Pi_{p}\left(\vec{V}_{h}, \xi, t\right) .
$$

Integrating (3.16) with the boundary condition (3.17), one finds

$$
p=c(h-\bar{\xi})+\Pi_{h}\left(\vec{V}_{h}, \xi, t\right)-\int_{\bar{\xi}}^{h} \Psi_{p}(\vec{V}, \vec{\xi}, t)
$$

If we consider that the derivatives of $h, V, \bar{V}$ are bounded and $\mathcal{O}(1)$, one can see that $p$ is determined up to order one and that

$$
p=c(h-\bar{\xi})-\bar{\kappa} \mathcal{H}+\mathcal{O}\left(\alpha+\frac{\theta_{R}}{\lambda} \beta\right)=p^{(0)}+\mathcal{O}\left(\alpha+\frac{\theta_{R}}{\lambda} \beta\right)
$$

Let us now see the influence of the scaling assumption $\varepsilon, \alpha, \beta, \delta \ll 1$ on the velocity profile. Expanding rescaled quantities in (3.5), (3.6) with respect to $\varepsilon, \alpha, \beta, \delta \ll 1$ and $\lambda, \bar{\kappa}=\mathcal{O}(1)$, we find for the rescaled quantities associated to the change of reference frame that

$$
\begin{aligned}
J & =\bar{J}\left(1-\varepsilon \theta_{R} \bar{\xi} \operatorname{tr}\left(\partial_{x} \mathbf{s}\right)\right)+\mathcal{O}\left(\varepsilon^{2} \theta_{R}^{2}\right), \\
\partial_{\xi} X & =\partial_{\xi} x-\varepsilon \theta_{R} \bar{\xi}\left(\partial_{\xi} \mathbf{s}\right)+\mathcal{O}\left(\varepsilon^{2} \theta_{R}^{2}\right), \\
M & =\bar{M}+2 \varepsilon \theta_{R} \bar{\xi}\left(\partial_{\xi} x\right)^{-1}\left(\left(\mathrm{Id}-\mathbf{s s}^{t}\right) \mathcal{H}_{b}\left(\mathrm{Id}-\mathbf{s} \mathbf{s}^{t}\right)\right)\left(\partial_{\xi} x\right)^{-t}+\mathcal{O}\left(\varepsilon^{2} \theta_{R}^{2}\right),
\end{aligned}
$$

with $\bar{J}=\frac{\operatorname{det}\left(\partial_{\xi} x\right)}{c}$ and $\bar{M}=\left(\partial_{\xi} x\right)^{-1}\left(\operatorname{Id}-\mathbf{s s}^{t}\right)\left(\partial_{\xi} x\right)^{-t}$. The rescaled quantities related to the flow unknows have the expansion

$$
\begin{aligned}
& \widetilde{h}=\bar{J} h-\varepsilon \theta_{R} \bar{J} \operatorname{tr}\left(\partial_{x} \mathbf{s}\right) \frac{h^{2}}{2}+\mathcal{O}\left(\varepsilon^{2} \theta_{R}^{2}\right) \\
& \bar{Z}=\bar{M}^{-1} \partial_{\bar{\xi}} V-2 \varepsilon \theta_{R}\left(\partial_{\xi} x\right)^{t} \mathcal{H}_{b}\left(\partial_{\xi} x\right) \bar{\xi}\left(\partial_{\bar{\xi}} V\right)+\mathcal{O}\left(\varepsilon^{2} \theta_{R}^{2}+\varepsilon^{2}\right),
\end{aligned}
$$

Note that from the expansion of $\widetilde{h}$, we can easily obtain an expansion of the physical fluid height $h$ :

$$
\bar{J} h=\widetilde{h}+\varepsilon \theta_{R} \operatorname{tr}\left(\partial_{x} \mathbf{s}\right) \frac{\widetilde{h}^{2}}{2 \bar{J}}+\mathcal{O}\left(\varepsilon^{2} \theta_{R}^{2}\right) .
$$


Now we insert the previous expansions into Equation (3.3) and find that

$$
\begin{aligned}
\partial_{\bar{\xi} \bar{\xi}}^{2}(\bar{J} V)-\lambda c \bar{J}\left(\partial_{\xi} x\right)^{-1} \mathbf{s}= & \beta \bar{J}\left(\partial_{t} V+\vec{V} \cdot \nabla_{\xi} V-\Gamma(\vec{V})\right)+\delta \bar{J} \bar{M} \nabla_{\xi} p \\
& -\delta \theta_{R} \bar{\xi} c \bar{J}\left(\partial_{\xi} x\right)^{-1}\left(\operatorname{tr}\left(\partial_{x} \mathbf{s}\right) I d+\partial_{x} \mathbf{s}\right) \mathbf{s} \\
& +\varepsilon \theta_{R} \bar{J}\left(\operatorname{tr}\left(\partial_{x} \mathbf{s}\right) \partial_{\bar{\xi}}\left(\bar{\xi} \partial_{\bar{\xi}} V\right)-2\left(\partial_{\xi} x\right)^{-1}\left(\partial_{x} \mathbf{s}\right)\left(\partial_{\xi} x\right) \partial_{\bar{\xi}} V\right) \\
& +2 \varepsilon \theta_{R} \bar{J}\left(\partial_{\xi} x\right)^{-1}\left(\partial_{x} \mathbf{s}\right)\left(\partial_{\xi} x\right) \partial_{\bar{\xi} \bar{\xi}}^{2}(V)+\mathcal{O}\left(\varepsilon^{2}+\varepsilon^{2} \theta_{R}^{2}\right) .
\end{aligned}
$$

In order to simplify the notation, we shall define the right hand side of (3.21) as $\Psi_{V}(\vec{V}, p, \vec{\xi}, t)$, and $(3.21)$ reads

$$
\partial_{\bar{\xi} \bar{\xi}}^{2}(\bar{J} V)-\lambda c \bar{J}\left(\partial_{\xi} x\right)^{-1} \mathbf{s}=\Psi_{V}(\vec{V}, p, \vec{\xi}, t) .
$$

The continuity of the fluid stress at the free boundary $\bar{\xi}=h$ implies (see Equation $(3.9))$ :

$$
\begin{aligned}
\bar{Z}_{h} & =\alpha \delta\left(\bar{P}_{h} M_{h}-\left(\bar{f}_{h}-\left(M_{h} \bar{Z}_{h}\right)^{t} \nabla_{\xi} h\right) \mathrm{Id}\right) \nabla_{\xi} h=\mathcal{O}\left(\varepsilon^{2}\right), \\
\partial_{\bar{\xi}} V_{h} & =2 \varepsilon \theta_{R}\left(\partial_{\xi} x\right)^{-1}\left(\partial_{x} \mathbf{s}\right)\left(\partial_{\xi} x\right) h \partial_{\bar{\xi}} V_{h}+\mathcal{O}\left(\varepsilon^{2}+\varepsilon^{2} \theta_{R}^{2}\right)=\Pi_{v}\left(\vec{V}_{h}, \xi, t\right) .
\end{aligned}
$$

The no-slip condition yields

$$
V_{0}=0 .
$$

Integrating (3.22) with respect to the cross-stream variable $\bar{\xi}$ with the boundary conditions $(3.23,3.24)$, one obtains

$$
V=-\lambda c\left(h \bar{\xi}-\frac{\bar{\xi}^{2}}{2}\right)\left(\partial_{\xi} x\right)^{-1} \mathbf{s}-\frac{1}{\bar{J}} \int_{0}^{\bar{\xi}} \int_{z}^{h} \Psi_{V}(\vec{V}, p, \vec{\xi}, t)+\bar{\xi} \Pi_{v}\left(\vec{V}_{h}, \xi, t\right) .
$$

It is easily seen that up to order one in $\varepsilon, \alpha, \beta, \delta$, the streamwise speed $V$ is uniquely defined by

$$
V=-\lambda c\left(h \bar{\xi}-\frac{\bar{\xi}^{2}}{2}\right)\left(\partial_{\xi} x\right)^{-1} \mathbf{s}+\mathcal{O}\left(\varepsilon \theta_{R}+\beta+\delta\right)=V^{(0)}+\mathcal{O}\left(\varepsilon \theta_{R}+\beta+\delta\right) .
$$

Up to order one, the profile of the streamwise speed $V$ is parabolic with respect to the variable $\bar{\xi}$; when the bottom surface is an inclined plane, this corresponds exactly to a Nusselt stationary flow. In the present case, the flow is locally close to a Nusslet type flow which is a local equilibrium. Thus, the scaling assumption $\varepsilon \ll 1$ implies that at each time, the solution is close to a local equilibrium; this is the other classical assumption made in the derivation of shallow water equations.

Finally, we obtain an asymptotic expansion for $\bar{V}$ by integration of the divergencefree condition $\nabla_{\xi} \cdot V+\partial_{\bar{\xi}} \bar{V}=0$ with the no-slip condition $\bar{V}(\xi, 0)=0$. One easily obtains

$$
\bar{V}=-\int_{0}^{\bar{\xi}} \nabla_{\xi} \cdot V=\bar{V}^{(0)}+\mathcal{O}\left(\beta+\delta+\varepsilon \theta_{R}\right),
$$


with $\bar{V}^{(0)}=-\int_{0}^{\bar{\xi}} \nabla_{\xi} \cdot V^{(0)}$.

We describe now the method to derive shallow water equations from the incompressible Navier-Stokes equations in the parameter regime described above; the method is mainly based on an iterative scheme. First, based on equations $(3.18,3.25$, 3.27 ), we define the sequence of functions $\left(V^{(n)}, \bar{V}^{(n)}, p^{(n)}\right)_{n \in \mathbb{N}}$ by

$$
\begin{aligned}
V^{(0)} & =-\lambda c\left(h \bar{\xi}-\frac{\bar{\xi}^{2}}{2}\right)\left(\partial_{\xi} x\right)^{-1} \mathbf{s}, \\
\bar{V}^{(0)} & =\int_{0}^{\bar{\xi}} \nabla_{\xi} \cdot V^{(0)} d z, \\
p^{(0)} & =c(h-\bar{\xi})-\bar{\kappa} \mathcal{H} .
\end{aligned}
$$

Then at the $(n+1)$-step, the functions $\left(V^{(n+1)}, \bar{V}^{(n+1)}, p^{(n+1)}\right)$ are defined as

$$
\begin{aligned}
V^{(n+1)} & =V^{(0)}-\frac{1}{\bar{J}} \int_{0}^{\bar{\xi}} \int_{z}^{h} \Psi_{V}\left(\vec{V}^{(n)}, p^{(n)}, \vec{\xi}, t\right) d s d z+\bar{\xi} \Pi_{v}\left(\vec{V}_{h}^{(n)}, \xi, t\right), \\
\bar{V}^{(n+1)} & =-\int_{0}^{\bar{\xi}} \nabla_{\xi} \cdot V^{(n+1)} d z, \\
p^{(n+1)} & =c(h-\bar{\xi})+\Pi_{h}\left(\vec{V}_{h}^{(n+1)}, \xi, t\right)-\int_{\bar{\xi}}^{h} \Psi_{p}\left(\vec{V}^{(n+1)}, \vec{\xi}, t\right) d z .
\end{aligned}
$$

Then, this sequence of functions converges formally to $(V, \bar{V}, p)$ a solution of the Navier-Stokes equations. More precisely, denote the operator $\mathcal{F}$ so that the iterative scheme define previously reads

$$
\left(V^{(n+1)}, \bar{V}^{(n+1)}, p^{(n+1)}\right)=\mathcal{F}\left(V^{(n)}, \bar{V}^{(n)}, p^{(n)}\right) .
$$

Then any solution $(V, \bar{V}, p)$ of the Navier Stokes equations with boundary conditions is a fixed point of $\mathcal{F}$. Moreover, this operator is $\mathcal{O}\left(\alpha+\beta+\delta+\varepsilon+\varepsilon \theta_{R}\right)$-Lipschitz on any bounded set. As a consequence, and provided that the sequence $\left(V^{(n)}, \bar{V}^{(n)}, p^{(n)}\right)$ and the derivatives of the Navier-Stokes solutions remain uniformly bounded with respect to $\alpha, \beta, \delta, \varepsilon, \varepsilon \theta_{R}$, one can prove by induction the following estimate

$$
\max \left(\left|V-V^{(n)}\right|,\left|\bar{V}-\bar{V}^{(n)}\right|,\left|p-p^{(n)}\right|\right)=\mathcal{O}\left(\left(\alpha+\beta+\delta+\varepsilon+\varepsilon \theta_{R}\right)^{n+1}\right) .
$$

As a consequence, we obtain an asymptotic expansion of $V, \bar{V}, p$ with respect to $\varepsilon, \alpha, \beta, \delta$ to any order in $\varepsilon, \alpha, \beta, \delta$. This step of the computation can be implemented using a formal computation software. Using this expansion, we easily deduce an expansion of $\tilde{v}$ and compute an expansion of the different terms in the average equation (3.13). Dropping terms in this equation of a fixed order, we obtain a hierarchy of models. In what follows, we compute the shallow water model obtained by dropping all small terms in the average equation. We shall see that an approximation of $V, p$ up to order one is needed to carry out such a computation. Let us first compute the zeroth order approximation of $V, p$ and see which terms we keep in (3.13). 
3.2.1. Asymptotic to order $\mathbf{0}$. In this section, we compute the zeroth order approximation of $V, p$ and check which terms we keep in (3.13). Recall that $V=V^{(0)}+\mathcal{O}\left(\beta+\delta+\epsilon \theta_{R}\right)$ with

$$
V^{(0)}=-\lambda c\left(h \bar{\xi}-\frac{\bar{\xi}^{2}}{2}\right)\left(\partial_{\xi} x\right)^{-1} \mathbf{s} .
$$

We can compute an approximation of $\widetilde{v}$ :

$$
\begin{aligned}
\widetilde{h} \widetilde{v}=\int_{0}^{h} J V & =\int_{0}^{h} \bar{J} V^{(0)}+\mathcal{O}\left(\varepsilon \theta_{R}+\beta+\delta\right) \\
& =-\lambda c \bar{J}\left(\partial_{\xi} x\right)^{-1} \mathbf{s} \int_{0}^{h}\left(h \bar{\xi}-\frac{\bar{\xi}^{2}}{2}\right)+\mathcal{O}\left(\varepsilon \theta_{R}+\beta+\delta\right) \\
& =-\lambda c \bar{J} \frac{h^{3}}{3}\left(\partial_{\xi} x\right)^{-1} \mathbf{s}+\mathcal{O}\left(\varepsilon \theta_{R}+\beta+\delta\right) .
\end{aligned}
$$

Then up to zeroth order, we find that

$$
\widetilde{v}=-\lambda c\left(\partial_{\xi} x\right)^{-1} \mathbf{s} \frac{\widetilde{h}^{2}}{3 \bar{J}^{2}}
$$

We calculate the zeroth order approximation of the other terms in the left hand side of (3.13) as functions of $\widetilde{h}, \widetilde{v}$.

The advection term $\int_{0}^{h} J V \otimes V$ reads:

$$
\begin{aligned}
\int_{0}^{h} J V \otimes V & =\bar{J} \int_{0}^{h} V^{(0)} \otimes V^{(0)}+\mathcal{O}\left(\varepsilon \theta_{R}+\beta+\delta\right) \\
& =\lambda^{2} c^{2} \bar{J}\left(\partial_{\xi} x\right)^{-1} \mathbf{s} \otimes\left(\partial_{\xi} x\right)^{-1} \mathbf{s} \int_{0}^{h}\left(h \bar{\xi}-\frac{\bar{\xi}^{2}}{2}\right)^{2}+\mathcal{O}\left(\varepsilon \theta_{R}+\beta+\delta\right) \\
& =\frac{2 \lambda^{2} c^{2}}{15} \frac{\widetilde{h}^{5}}{\bar{J}^{4}}\left(\partial_{\xi} x\right)^{-1} \mathbf{s} \otimes\left(\partial_{\xi} x\right)^{-1} \mathbf{s}+\mathcal{O}\left(\varepsilon \theta_{R}+\beta+\delta\right) \\
& =\frac{6}{5} \widetilde{h} \widetilde{v} \otimes \widetilde{v}+\mathcal{O}\left(\varepsilon \theta_{R}+\beta+\delta\right) .
\end{aligned}
$$

Integrating the pressure equation (3.16) with the boundary condition (3.17), one proves that the pressure $p$ is given by

$$
p=-\bar{\kappa} \mathcal{H}+c(h-\bar{\xi})+\mathcal{O}\left(\varepsilon \theta_{R}+\alpha\right) .
$$

Up to zeroth order, the average terms containing the pressure are given by:

$$
\begin{aligned}
& \int_{0}^{h} J p M=\bar{J} \bar{M}\left(c \frac{h^{2}}{2}-\bar{\kappa} \mathcal{H} h\right), \\
& \int_{0}^{h} p \nabla_{\xi} \cdot(J M)=\nabla_{\xi} \cdot(\bar{J} \bar{M})\left(c \frac{h^{2}}{2}-\bar{\kappa} \mathcal{H} h\right) .
\end{aligned}
$$


As a consequence, the average equation (3.13) reads:

$$
\begin{aligned}
& \partial_{t}(\widetilde{h} \widetilde{v})+\frac{6}{5} \nabla_{\xi} \cdot(\widetilde{h} \widetilde{v} \otimes \widetilde{v})+\frac{\delta}{\beta} \bar{J} \bar{M} \nabla_{\xi}\left(c \frac{\widetilde{h}^{2}}{2 \bar{J}}\right)-\bar{\kappa} \frac{\delta}{\beta} \widetilde{h} \bar{M} \nabla_{\xi} \mathcal{H} \\
= & -\frac{1}{\beta}\left(\lambda c \int_{0}^{h} J\left(\partial_{\xi} X\right)^{-1} \mathbf{s}+\bar{J} \partial_{\bar{\xi}} V(0)\right) \\
& +\int_{0}^{h} J\left(\partial_{\xi} X\right)^{-1}\left(\theta_{R} \bar{\Gamma}(\vec{V}) \mathbf{s}-\left(\partial_{\xi \xi}^{2} X\right) \cdot V \cdot V\right) \\
& -\frac{1}{\beta} \int_{0}^{h} J\left(\partial_{\bar{\xi}} M\right) \bar{Z}+\mathcal{O}\left(\left(\varepsilon \theta_{R}+\alpha\right) \frac{\delta}{\beta}+\beta+\delta+\varepsilon \theta_{R}\right) .
\end{aligned}
$$

It is a straightforward computation to prove that the zeroth order terms in the right hand side of (3.37) read

$$
\begin{aligned}
& \int_{0}^{h} J\left(\partial_{\xi} X\right)^{-1}\left(\theta_{R} \bar{\Gamma}(\vec{V}) \mathbf{s}-\left(\partial_{\xi \xi}^{2} X\right) \cdot V \cdot V\right) \\
= & -\frac{2 \widetilde{h}^{5}}{15 \bar{J}^{4}} \lambda^{2} c^{2}\left(\partial_{\xi} x\right)^{-1}\left(\left(\partial_{\xi \xi}^{2} x\right) \cdot\left(\partial_{\xi} x\right)^{-1} \mathbf{s} \cdot\left(\partial_{\xi} x\right)^{-1} \mathbf{s}+\theta_{R}\left(\mathbf{s}^{t} \mathcal{H}_{b} \mathbf{s}\right) \mathbf{s}\right)+\mathcal{O}\left(\varepsilon \theta_{R}+\beta+\delta\right), \\
& \frac{1}{\beta} \int_{0}^{h} J\left(\partial_{\bar{\xi}} M\right) \bar{Z}=-\frac{\delta}{\beta} c\left(\partial_{\xi} x\right)^{-1}\left(\partial_{x} \mathbf{s}\right) \mathbf{s} \frac{\widetilde{h}^{2}}{\bar{J}}+\mathcal{O}\left(\frac{\varepsilon \theta_{R}}{\beta}\left(\delta+\delta \theta_{R}+\beta+\varepsilon \theta_{R}\right)\right) .
\end{aligned}
$$

Due to the presence of the factor $-\frac{1}{\beta}$ in front of $\lambda c \int_{0}^{h} J\left(\partial_{\xi} X\right)^{-1} \mathbf{s}+\bar{J} \partial_{\bar{\xi}} V(0)$, we need an expansion of the wall shear $\partial_{\bar{\xi}} V(0)$ and the average velocity $\widetilde{v}$ up to order 1 in order to eliminate $\partial_{\bar{\xi}} V(0)$ from (3.37). This is done in the next section.

3.2.2. Asymptotic to order 1 . This section is devoted to the computation of an asymptotic expansion of $V$ up to order one: this enables us to compute $\widetilde{v}$ and $\partial_{\bar{\xi}} V(0)$ as a function of $\widetilde{h}, \xi$ and $\varepsilon, \alpha, \beta, \delta$ up to order one. We can then insert the resulting expressions in (3.37); dropping small terms, we find a shallow water model for arbitrary topography.

We first prove that

$$
\lambda c \int_{0}^{h} J\left(\partial_{\xi} X\right)^{-1} \mathbf{s}=\lambda c \widetilde{h}\left(\partial_{\xi} x\right)^{-1} \mathbf{s}+\delta \theta_{R} c \frac{\widetilde{h}^{2}}{2 \bar{J}}\left(\partial_{\xi} x\right)^{-1}\left(\partial_{x} \mathbf{s}\right) \mathbf{s}+\mathcal{O}\left(\varepsilon^{2} \theta_{R}^{2}\right) .
$$

Using the iterative scheme described in the previous section, we find after an integration on $(\bar{\xi}, h)$ and up to order one:

$$
\begin{aligned}
& \partial_{\bar{\xi}}(\bar{J} V)+\lambda c \bar{J}\left(\partial_{\xi} x\right)^{-1} \mathbf{s}(h-\bar{\xi}) \\
= & \bar{J} \int_{\bar{\xi}}^{h} \beta\left(\Gamma\left(\vec{V}^{(0)}\right)-\partial_{t} V^{(0)}-\vec{V}^{(0)} \cdot \nabla_{\vec{\xi}} V^{(0)}\right) \\
& -\delta \bar{M} \nabla_{\xi} p^{(0)}+\delta \theta_{R} c \bar{J}\left(\partial_{\xi} x\right)^{-1}\left(\operatorname{tr}\left(\partial_{x} \mathbf{s}\right) I d+\partial_{x} \mathbf{s}\right) \mathbf{s} \int_{\bar{\xi}}^{h} z d z \\
& +\varepsilon \theta_{R} \bar{J} \operatorname{tr}\left(\partial_{x} \mathbf{s}\right) \bar{\xi}\left(\partial_{\bar{\xi}} V\right)^{(0)}+2 \varepsilon \theta_{R} \bar{J}\left(\partial_{\xi} x\right)^{-1}\left(\partial_{x} \mathbf{s}\right)\left(\partial_{\xi} x\right) V^{(0)} \\
& +2 \varepsilon \theta_{R} \bar{J}\left(\partial_{\xi} x\right)^{-1}\left(\partial_{x} \mathbf{s}\right)\left(\partial_{\xi} x\right) \partial_{\bar{\xi}}\left(\bar{\xi} V^{(0)}\right) \\
& +\mathcal{O}\left(\varepsilon^{2} \theta_{R}^{2}+\beta\left(\beta+\delta+\varepsilon \theta_{R}\right)+\delta\left(\alpha+\varepsilon \theta_{R}\right)\right) .
\end{aligned}
$$


Setting $\bar{\xi}=0$ in (3.38), one obtains an expansion for $\partial_{\bar{\xi}} V(0)$ :

$$
\bar{J} \partial_{\bar{\xi}} V(0)+\lambda c \widetilde{h}\left(\partial_{\xi} x\right)^{-1} \mathbf{s}=\delta P_{1}+\beta \lambda P_{2}+P_{3},
$$

with $P_{i}$ defined by

$$
\begin{aligned}
P_{1}= & \theta_{R} c\left(\partial_{\xi} x\right)^{-1}\left(\partial_{x} \mathbf{s}\right) \mathbf{s} \frac{\widetilde{h}^{2}}{2 \bar{J}}-\bar{J} \bar{M}\left(\nabla_{\xi}(c h-\bar{\kappa} \mathcal{H}) h-\nabla_{\xi} c \frac{h^{2}}{2}\right), \\
P_{2}= & \bar{J} c\left(\partial_{\xi} x\right)^{-1} \mathbf{s} h_{t} \frac{h^{2}}{2}-\lambda c \nabla_{\xi} \cdot\left(\bar{J} c\left(\partial_{\xi} x\right)^{-1} \mathbf{s}\right) \frac{h^{5}}{120}+\lambda c \nabla_{\xi} \cdot\left(\bar{J} c h\left(\partial_{\xi} x\right)^{-1} \mathbf{s}\right) \frac{h^{4}}{120} \\
& -\bar{J} \lambda c^{2}\left(\partial_{\xi} x\right)^{-1}\left(\left(\partial_{\xi \xi}^{2} x\right) \cdot\left(\partial_{\xi} x\right)^{-1} \mathbf{s} \cdot\left(\partial_{\xi} x\right)^{-1} \mathbf{s}+\theta_{R}\left(\mathbf{s}^{t} \mathcal{H}_{b} \mathbf{s}\right) \mathbf{s}\right) \frac{2 h^{5}}{15} \\
& -\lambda c \bar{J}\left(\partial_{\xi} x\right)^{-1} \mathbf{s} \cdot \nabla_{\xi}\left(c h\left(\partial_{\xi} x\right)^{-1} \mathbf{s}\right) \frac{5 h^{4}}{24}+\lambda c \bar{J}\left(\partial_{\xi} x\right)^{-1} s \cdot \nabla_{\xi}\left(c\left(\partial_{\xi} x\right)^{-1} \mathbf{s}\right) \frac{3 h^{5}}{40}, \\
P_{3}= & \mathcal{O}\left(\varepsilon^{2} \theta_{R}^{2}+\beta\left(\beta+\delta+\varepsilon \theta_{R}\right)+\delta\left(\alpha+\varepsilon \theta_{R}\right)\right) .
\end{aligned}
$$

Let us now compute an asymptotic expansion for $\widetilde{h} \widetilde{v}$ up to order one.

Integrating twice Equation (3.38) on $\partial_{\bar{\xi}} V$, we can prove that

$$
\int_{0}^{h} \bar{J} V+\lambda c \bar{J}\left(\partial_{\xi} x\right)^{-1} \mathbf{s} \frac{h^{3}}{3}=\delta Q_{1}+\beta \lambda Q_{2}+Q_{3},
$$

with $Q_{i}$ given by

$$
\begin{aligned}
Q_{1}= & \theta_{R} c \bar{J}\left(\partial_{\xi} x\right)^{-1}\left(\partial_{x} \mathbf{s}\right) \mathbf{s} \frac{h^{4}}{4}-\theta_{R} c \bar{J}\left(\partial_{\xi} x\right)^{-1}\left(\partial_{x} \mathbf{s}\right) \mathbf{s} \frac{5 h^{4}}{12} \\
& +\theta_{R} \bar{J} c\left(\partial_{\xi} x\right)^{-1}\left(\operatorname{tr}\left(\partial_{x} \mathbf{s}\right) I d+\left(\partial_{x} \mathbf{s}\right)\right) \mathbf{s} \frac{5 h^{4}}{24}-\theta_{R} c \bar{J} \operatorname{tr}\left(\partial_{x} \mathbf{s}\right)\left(\partial_{\xi} x\right)^{-1} \mathbf{s} \frac{h^{4}}{12} \\
& -\bar{J} \bar{M}\left(\nabla_{\xi}(c h-\bar{\kappa} \mathcal{H}) \frac{h^{3}}{3}-\nabla_{\xi} c \frac{5 h^{4}}{24}\right), \\
Q_{2}= & c \bar{J}\left(\partial_{\xi} x\right)^{-1} \mathbf{s} h_{t} \frac{5 h^{4}}{24} \\
& -\bar{J} \lambda c^{2}\left(\partial_{\xi} x\right)\left(\left(\partial_{\xi \xi}^{2} x\right) \cdot\left(\partial_{\xi} x\right)^{-1} \mathbf{s} \cdot\left(\partial_{\xi} x\right)^{-1} \mathbf{s}+\theta_{R}\left(\mathbf{s}^{t} \mathcal{H}_{b} \mathbf{s}\right) \mathbf{s}\right) \frac{2 h^{7}}{35} \\
& -\lambda c \bar{J}\left(\partial_{\xi} x\right)^{-1} \mathbf{s} \cdot \nabla_{\xi}\left(c h \partial_{\xi} x^{-1} \mathbf{s}\right) \frac{11 h^{6}}{120}+\lambda c \bar{J}\left(\partial_{\xi} x\right)^{-1} \mathbf{s} \cdot \nabla_{\xi}\left(c\left(\partial_{\xi} x\right)^{-1} \mathbf{s}\right) \frac{29 h^{7}}{840} \\
& +\lambda c \nabla_{\xi} \cdot\left(\bar{J} c h\left(\partial_{\xi} x\right)^{-1} \mathbf{s}\right)\left(\partial_{\xi} x\right)^{-1} \mathbf{s} \frac{h^{6}}{60}-\lambda c \nabla_{\xi} \cdot\left(\bar{J} c\left(\partial_{\xi} x\right)^{-1} \mathbf{s}\right)\left(\partial_{\xi} x\right)^{-1} \mathbf{s} \frac{3 h^{7}}{840} \\
Q_{3}= & \mathcal{O}\left(\varepsilon^{2} \theta_{R}^{2}+\beta\left(\beta+\delta+\varepsilon \theta_{R}\right)+\delta\left(\alpha+\varepsilon \theta_{R}\right)\right) .
\end{aligned}
$$

Moreover, it is easily seen that we can deduce $\widetilde{h} \widetilde{v}$ from $\int_{0}^{h} \bar{J} V d \bar{\xi}$ with the equation:

$$
\widetilde{h} \widetilde{v}=\int_{0}^{h} \bar{J} V-\varepsilon \theta_{R} \int_{0}^{h} \operatorname{tr}\left(\partial_{x} \mathbf{s}\right) \bar{\xi} V^{(0)}+\mathcal{O}\left(\varepsilon \theta_{R}\left(\beta+\delta+\epsilon \theta_{R}\right)\right) .
$$

Finally we have

$$
\lambda c \bar{J}\left(\partial_{\xi} x\right)^{-1} \mathbf{s} \frac{h^{3}}{3}=\lambda c\left(\partial_{\xi} x\right)^{-1} \mathbf{s} \frac{\widetilde{h}^{3}}{3 \bar{J}^{2}}+\delta \theta_{R} c \operatorname{tr}\left(\partial_{x} \mathbf{s}\right)\left(\partial_{\xi} x\right)^{-1} \mathbf{s} \frac{\widetilde{h}^{4}}{2 \bar{J}^{2}}+\mathcal{O}\left(\varepsilon^{2} \theta_{R}^{2}\right) .
$$


Using equations (3.41), (3.43) and (3.44), one finds an expansion for $\widetilde{h} \widetilde{v}$ up to order one as a function of $\widetilde{h}, \xi$ and $(\varepsilon, \alpha, \delta \beta)$. Similarly, Equation (3.39) gives an expansion of $\partial_{\bar{\xi}} V(0)$ up to order one; thus we can eliminate $\partial_{\bar{\xi}} V(0)$ from Equation (3.37) to obtain a shallow water model. We shall see later that there are different ways of eliminating $\partial_{\bar{\xi}} V(0)$, which gives a family of models. More precisely, from (3.41), (3.43), (3.44) and (3.39), we define $R_{1}$ and $R_{2}$ to be the terms of order one such that

$$
\begin{aligned}
\bar{J} \partial_{\bar{\xi}} V(0)= & -\lambda c \widetilde{h}\left(\partial_{\xi} x\right)^{-1} \mathbf{s}-R_{1}(\widetilde{h}, \xi) \\
& +\mathcal{O}\left(\varepsilon^{2} \theta_{R}^{2}+\beta\left(\beta+\delta+\varepsilon \theta_{R}\right)+\delta\left(\alpha+\varepsilon \theta_{R}\right)\right), \\
\widetilde{h} \widetilde{v}= & \lambda c \frac{\widetilde{h}^{3}}{3 \bar{J}^{2}}\left(\partial_{\xi} x\right)^{-1} \mathbf{s}-R_{2}(\widetilde{h}, \xi) \\
& +\mathcal{O}\left(\varepsilon^{2} \theta_{R}^{2}+\beta\left(\beta+\delta+\varepsilon \theta_{R}\right)+\delta\left(\alpha+\varepsilon \theta_{R}\right)\right) .
\end{aligned}
$$

From (3.45), we compute an expansion of $\partial_{\bar{\xi}} V(0)$ up to order one involving the average quantities $\widetilde{h}, \widetilde{v}$, the variable $\xi$ and the parameters $\alpha, \beta, \delta, \varepsilon$ :

$$
\begin{aligned}
-\bar{J} \partial_{\bar{\xi}} V(0)= & -\frac{3 \bar{J}^{2}}{\widetilde{h}} \widetilde{v}+R_{1}(\widetilde{h}, \xi)-\frac{3 \bar{J}^{2}}{\widetilde{h}^{2}} R_{2}(\widetilde{h}, \xi) \\
& +\mathcal{O}\left(\varepsilon^{2} \theta_{R}^{2}+\beta\left(\beta+\delta+\varepsilon \theta_{R}\right)+\delta\left(\alpha+\varepsilon \theta_{R}\right)\right)
\end{aligned}
$$

Inserting (3.46) into (3.37), one finds

$$
\begin{aligned}
\partial_{t} \widetilde{h}+ & \nabla_{\xi} \cdot(\widetilde{h} \widetilde{v})=0 \\
& \partial_{t}(\widetilde{h} \widetilde{v})+\frac{6}{5} \nabla_{\xi} \cdot(\widetilde{h} \widetilde{v} \otimes \widetilde{v})+\frac{\delta}{\beta} \overline{J M} \nabla_{\xi}\left(c \frac{\widetilde{h}^{2}}{2 \bar{J}}\right)-\bar{\kappa} \frac{\delta}{\beta} \widetilde{h} \bar{M} \nabla_{\xi} \mathcal{H} \\
= & -\frac{1}{\beta}\left(\lambda c \widetilde{h}\left(\partial_{\xi} x\right)^{-1} \mathbf{s}+\frac{3 \bar{J}^{2}}{\widetilde{h}} \widetilde{v}\right)+\mathcal{T}_{1}+\mathcal{R},
\end{aligned}
$$

with

$$
\mathcal{R}=\mathcal{O}\left(\frac{1}{\beta}\left(\varepsilon^{2} \theta_{R}^{2}+\delta\left(\alpha+\varepsilon \theta_{R}\right)+\beta+\delta+\varepsilon \theta_{R}\right) .\right.
$$

The function $\mathcal{T}_{1}$ depends on $\xi$ and $\widetilde{h}$ and reads:

$$
\mathcal{T}_{1}=\frac{\delta}{\beta} \mathcal{T}_{1}^{(1)}+\lambda^{2} \mathcal{T}_{1}^{(2)}
$$


with

$$
\begin{aligned}
\mathcal{T}_{1}^{(1)}= & \left(\frac{\bar{M}}{8} \nabla_{\xi} c+\frac{3}{4} c\left(\partial_{\xi} x\right)^{-1}\left(\partial_{x} \mathbf{s}\right) \mathbf{s}-\frac{5}{8} c\left(\partial_{\xi} x\right)^{-1}\left(\partial_{x} \mathbf{s}\right) \mathbf{s}-\frac{c}{2} \operatorname{tr}\left(\partial_{x} \mathbf{s}\right)\left(\partial_{\xi} x\right)^{-1} \mathbf{s}\right) \frac{\widetilde{h}^{2}}{\bar{J}} \\
\mathcal{T}_{1}^{(2)}= & c\left(\partial_{\xi} x\right)^{-1} \mathbf{s} . \nabla_{\xi}\left(c\left(\partial_{\xi} x\right)^{-1} \mathbf{s}\right) \frac{\widetilde{h}^{5}}{35 \bar{J}^{4}}-c\left(\partial_{\xi} x\right)^{-1} \mathbf{s} . \nabla_{\xi}\left(c \frac{\widetilde{h}}{\bar{J}}\left(\partial_{\xi} x\right)^{-1} \mathbf{s}\right) \frac{\widetilde{h}^{4}}{1 \bar{J}^{4}} \\
& +\left(\nabla_{\xi} \cdot\left(c \frac{\widetilde{h}^{3}}{\bar{J}^{3}}\left(\partial_{\xi} x\right)^{-1} \mathbf{s}\right) \frac{\widetilde{h}^{2}}{24 \bar{J}^{2}}+\nabla_{\xi} \cdot\left(c \tilde{h}\left(\partial_{\xi} x\right)^{-1} \mathbf{s}\right) \frac{\widetilde{h}^{4}}{120 \bar{J}^{4}}\right) c\left(\partial_{\xi} x\right)^{-1} \mathbf{s} \\
& -\nabla_{\xi} \cdot\left(\bar{J} c\left(\partial_{\xi} x\right)^{-1} \mathbf{s}\right) \frac{\widetilde{h}^{5}}{420 \bar{J}^{5}} c\left(\partial_{\xi} x\right)^{-1} \mathbf{s} \\
& -\frac{6}{35} c^{2}\left(\partial_{\xi} x\right)^{-1}\left(\partial_{\xi \xi}^{2} x \cdot\left(\partial_{\xi} x\right)^{-1} \mathbf{s} .\left(\partial_{\xi} x\right)^{-1} \mathbf{s}+\theta_{R}\left(\mathbf{s}^{t} \mathcal{H}_{b} \mathbf{s}\right) \mathbf{s}\right) \frac{\widetilde{h}^{5}}{\bar{J}^{4}} .
\end{aligned}
$$

We easily see that provided that the dimensionless numbers $R e, F, \bar{\kappa}$ are of order one, the higher order term $\mathcal{R}$ is $\mathcal{O}(\varepsilon)$ and it is negligible in the scaling limit $\varepsilon \ll 1$. Then dropping these high order terms, one finds a shallow water model for $\widetilde{h}, \widetilde{h} \widetilde{v}$ in a closed form. Furthermore, we clearly see that the conclusion of the expansion remains true if we only suppose that $\alpha, \beta, \delta, \varepsilon \theta_{R} \ll 1$ and $\frac{\varepsilon^{2} \theta_{R}^{2}}{\beta}, \frac{\delta}{\beta} \alpha, \frac{\delta}{\beta} \varepsilon \theta_{R} \ll 1$. As a consequence, the model remains vallid not only for Reynolds and Froude numbers of order one but also for $\mathbf{R e}, \mathbf{F} \rightarrow \infty$ as $\varepsilon \rightarrow 0$ provided that we choose a suitable dependence of $\mathbf{R e}, \mathbf{F}$ with respect to $\varepsilon$.

This formulation has the advantage of being independent of the parametrization of the bottom surface $\mathcal{S}$, but it is quite hard to work with that formulation. In order to carry out numerical simulations, we have to specify curvilinear coordinates. In the following, we are going to choose particular parametrizations of the bottom surface $\mathcal{S}$. Moreover, we shall be more precise on the asymptotic expansion of the mean curvature of the free surface $\mathcal{H}$ with respect to $\varepsilon, \xi$ and $\widetilde{h}, \mathcal{H}_{b}$; this will be done in the next section for a particular parametrization.

\section{Shallow water models for particular parametrizations}

In this section, we parametrize the bottom surface with two systems of coordinates: the "steepest descent" curvilinear coordinates and the classical orthonormal coordinates. We write the shallow water equations in this framework for $n$-dimensional flows $(n=2$ or $n=3)$, which gives $(n-1)$-dimensional shallow water equations.

4.1. Shallow water equations in the steepest descent parametrization. In what follows, we describe the "steepest descent" curvilinear coordinates and write the shallow water equations in that setting. First, we define $\theta, \phi$ such that the normal $\mathbf{n}$ to the surface $\mathcal{S}$ is given by (see part 2.1):

$$
\mathbf{n}=\left(\begin{array}{c}
-\frac{\nabla_{x} z}{\sqrt{1+\left\|\nabla_{x} z\right\|^{2}}} \\
\frac{1}{\sqrt{1+\left\|\nabla_{x} z\right\|^{2}}}
\end{array}\right) \quad \Longleftrightarrow \mathbf{n}=\left(\begin{array}{c}
\sin (\theta) \cos (\phi) \\
\sin (\theta) \sin (\phi) \\
\cos (\theta)
\end{array}\right)
$$


Here $\theta$ represents the local inclination of the bottom surface. We are going to use the "steepest descent" curvilinear coordinates $\xi_{1}, \xi_{2}$ so that we have

$$
\begin{aligned}
\frac{\partial x}{\partial \xi_{1}} & =\cos (\theta) \cos (\phi), \frac{\partial x}{\partial \xi_{2}}=-\sin (\phi), \\
\frac{\partial y}{\partial \xi_{1}} & =\cos (\theta) \sin (\phi), \frac{\partial y}{\partial \xi_{2}}=\cos (\phi),
\end{aligned}
$$

or equivalently

$$
\partial_{\xi} x=\left(\begin{array}{cc}
\cos (\theta) \cos (\phi) & -\sin (\phi) \\
\cos (\theta) \sin (\phi) & \cos (\phi)
\end{array}\right) .
$$

This system of coordinates is uniquely defined provided that a "steepest descent" direction exists; this condition is satisfied if $\mathbf{s} \neq 0$. This direction is at the intersection of the tangent plane to the bottom surface and the plane spanned by the normal to the surface and the gravity vector; we shall see later that it is the principal direction where the fluid flows. As a consequence of this definition, we see that this system of coordinates is designed for gravity-driven flows but is not relevant for fluids driven by other external forces. In this system of coordinates, the rescaled quantities $\bar{J}, \bar{M}$ associated to the change of reference frame are particularly simple. It is easily proved that

$$
\bar{J}=1, \quad \bar{M}=\mathrm{Id}, \quad c\left(\partial_{\xi} x\right)^{-1} \mathbf{s}=-\sin (\theta) \mathbf{e}_{1},
$$

with $\mathbf{e}_{1}=(1,0)^{t}$.

Let us now compute an asymptotic expansion for the rescaled curvature of the free surface. Using Lem. 2.2, the rescaled unitary normal $\vec{N}$ to the free surface reads $\vec{N}=\frac{\vec{n}}{\|\vec{n}\|}$ with

$$
\vec{n}=\left(\begin{array}{c}
-\mathbf{s}-\varepsilon\left(\mathrm{Id}-\mathbf{s s}^{t}\right)\left(\partial_{\xi} x\right)^{-t} \nabla_{\xi} h \\
c-\varepsilon\left(c\left(\partial_{\xi} x\right)^{-1} \mathbf{s}\right)^{t} \nabla_{\xi} h
\end{array}\right)+\mathcal{O}\left(\varepsilon^{2} \theta_{R}\right) .
$$

Then the rescaled mean curvature is given by

$$
\begin{aligned}
(n-1) \mathcal{H} & =\operatorname{div}_{x}\left(\left(\mathbf{s} \varepsilon\left(\mathrm{Id}-\mathbf{s s}^{t}\right)\left(\partial_{\xi} x\right)^{-t} \nabla_{\xi} h\right) /\|\vec{n}\|\right), \\
& =\theta_{R} \operatorname{tr}\left(\left(\partial_{\xi} x\right)^{-1} \partial_{\xi} \mathbf{s}\right)+\varepsilon \operatorname{tr}\left(\left(\partial_{\xi} x\right)^{-1} \partial_{\xi}\left(\left(\partial_{\xi} x\right) \nabla_{\xi} h\right)\right)+\mathcal{O}\left(\varepsilon^{2}\right) .
\end{aligned}
$$

As a consequence, the expansion of $\mathcal{H}$ with respect to $\varepsilon$ is given by

$$
\mathcal{H}=\theta_{R} H_{b}+\frac{\varepsilon}{n-1}\left(\Delta_{\xi} \widetilde{h}+\theta_{R} \nabla_{\xi} \widetilde{h} \cdot V_{\theta, \phi}\right)+O\left(\varepsilon^{2}\right),
$$

with $V_{\theta, \phi}=\left(\cos (\theta) \sin (2 \phi) \partial_{2} \phi-\tan (\theta) \partial_{1} \theta,-\frac{\partial_{1} \phi}{\cos (\theta)}\right)^{t}$.

Here, $\theta_{R} H_{b}$ represents the mean curvature of the bottom surface. At this stage, two situations occur. Either $\theta_{R}=\mathcal{O}(1)$ or $\theta_{R}=\prime(\varepsilon)$ : in the first case the mean curvature of the free surface is, up to order one, given by the mean curvature of the bottom surface:

$$
\mathcal{H}=-\frac{1}{2}\left(\frac{\partial \theta}{\partial \xi_{1}}+\sin (\theta) \frac{\partial \phi}{\partial \xi_{2}}\right)+\mathcal{O}(\varepsilon)
$$


In this particular case, the shallow water equations (3.47) have the more explicit form:

$$
\begin{aligned}
\partial_{t} \widetilde{h}+\nabla_{\xi} \cdot(\widetilde{h} \widetilde{v})= & 0, \\
& \partial_{t}(\widetilde{h} \widetilde{v})+\nabla_{\xi} \cdot\left(\frac{6}{5} \widetilde{h} \widetilde{v} \widetilde{v}-\frac{\lambda^{2} \sin ^{2}(\theta) \widetilde{h}^{5}}{75} \mathbf{e}_{1} \otimes \mathbf{e}_{1}\right)+\frac{\delta}{2 \beta} \nabla_{\xi}\left(\cos (\theta) \widetilde{h}^{2}\right) \\
= & -\frac{\bar{\kappa} \delta \theta_{R}}{2 \beta} \widetilde{h} \nabla_{\xi} \cdot\left(\partial_{1} \theta+\sin (\theta) \partial_{2} \phi\right)+\frac{1}{\beta}\left(\lambda \widetilde{h} \sin (\theta) \mathbf{e}_{1}-\frac{3 \widetilde{v}}{\widetilde{h}}\right) \\
& +\frac{\delta}{\beta} \widetilde{h}^{2} \sin (\theta)\left(\begin{array}{c}
-\frac{\theta_{R}}{2}\left(\partial_{1} \theta+\sin (\theta) \partial_{2} \phi\right)+\frac{\theta_{R}-1}{8} \partial_{1} \phi \\
-\frac{1}{8}\left(\partial_{2} \theta-\theta_{R} \sin (\theta) \partial_{1} \phi\right)
\end{array}\right) \\
& -\frac{6}{35} \lambda^{2} \widetilde{h}^{5} \sin ^{2}(\theta)\left(\begin{array}{c}
\left(\theta_{R}-1\right) \tan (\theta) \partial_{1} \theta \\
\cos (\theta) \phi_{1}
\end{array}\right) \\
& -\frac{3}{175} \lambda^{2} \widetilde{h}^{5} \sin (\theta) \cos (\theta) \partial_{1} \theta \mathbf{e}_{1} .
\end{aligned}
$$

If we restrict our attention to a 2-dimensional flow for the Navier-Stokes system and $\theta_{R}=1$, we easily get the one dimensional shallow water model

$$
\begin{aligned}
& \partial_{t} \widetilde{h}+\partial_{\xi}(\widetilde{h} \widetilde{v})=0, \\
& \partial_{t}(\widetilde{h} \widetilde{v})+\partial_{\xi}\left(\frac{6}{5} \widetilde{h} \widetilde{v}^{2}-\frac{\lambda^{2} \sin ^{2}(\theta) \widetilde{h}^{5}}{75}+\frac{\delta \cos (\theta) \widetilde{h}^{2}}{2 \beta}\right)+\bar{\kappa} \frac{\delta}{\beta} \widetilde{h} \partial_{\xi \xi}^{2} \theta \\
= & \frac{1}{\beta}\left(\lambda \widetilde{h} \sin (\theta)-\frac{3 \widetilde{v}}{\widetilde{h}}\right)-\left(\frac{\delta}{2 \beta}+\frac{3 \lambda^{2} \widetilde{h}^{3}}{175} \cos (\theta)\right) \sin (\theta) \partial_{\xi} \theta .
\end{aligned}
$$

Here the $\xi$ variable is the classical curvilinear coordinate. We clearly see that no contribution including third order derivatives of $\widetilde{h}$ appears in the capillary term. In this case, the capillary effects are essentially supported by the curvature of the bottom surface which is assumed to be not small: capillarity is not dispersive in this case and acts as a classical friction or damping term $-\kappa \widetilde{h} \nabla_{\xi} H_{b}$, depending on the mean curvature sign of the bottom surface.

In order to recover the classical "dispersive" capillary term involving third order derivatives of $\widetilde{h}$, let us assume that the curvature of the bottom is small: more precisely we consider $\theta_{R}=\varepsilon \widetilde{\theta}_{R}$. In this particular case, we find:

$$
\mathcal{H}=\varepsilon \widetilde{\theta}_{R} H_{b}+\frac{\varepsilon}{n-1} \Delta_{\xi} \widetilde{h}+O\left(\varepsilon^{2}\right)
$$

Then, the contribution of the capillarity term in (3.47) is negligible if $\bar{\kappa}=\mathcal{O}(1)$ : we only see the influence of the capillary terms provided that $\varepsilon \bar{\kappa}=\mathcal{O}(1)$. Denoting $\widetilde{\kappa}=\varepsilon \bar{\kappa}$, the capillary term in (3.47) reads:

$$
-\bar{\kappa} \frac{\delta}{\beta} h \nabla_{\xi} \mathcal{H}=-\widetilde{\kappa} \frac{\delta}{\beta} \widetilde{h} \nabla_{\xi}\left(\widetilde{\theta}_{R} H_{b}+\frac{1}{n-1} \Delta_{\xi} \widetilde{h}\right)+\mathcal{O}(\varepsilon)
$$

In the case of shallow water flows over a flat and horizontal bottom, we clearly recover the classical form of the capillary term $-\widetilde{\kappa} \frac{\delta}{\beta} \widetilde{h} \nabla \Delta \widetilde{h}$ found in the literature; see $[3,4]$ for a mathematical analysis of shallow water models with this kind of capillary term and references therein.

4.2. Shallow water equation in orthogonal coordinates. In the literature, the shallow water equations or lubrication model over varying topographies are usually 
written using orthogonal curvilinear coordinates. In order to compare our results with several shallow water equations obtained previously $[9,10]$, we write the shallow water equations using orthogonal coordinates. In this setting, the basis vectors of the tangent plane to the bottom surface are directed along the directions of maximal and minimal curvatures.

Define $m_{i}=\left\|\frac{\partial x(\xi)}{\partial \xi_{i}}\right\|$ and $h_{i}=m_{i}\left(1-\varepsilon \theta_{R} k_{i} \bar{\xi}\right)$, where $k_{i}$ is the mean curvature of the bottom in the direction $\xi_{i}$. In order to simplify the discussion, let us choose an orthogonal system so that $m_{i}=1$. Then, if we denote $\mathbf{n}=$ $(\sin (\theta) \cos (\phi), \sin (\theta) \cos (\phi), \cos (\theta))$, we easily establish that

$$
\bar{M}=I d, \quad \bar{J}=1, \quad c\left(\partial_{\xi} x\right)^{-1} \mathbf{s}=-\sin (\theta)\left(\begin{array}{c}
\cos (\phi) \\
\sin (\phi)
\end{array}\right)=-\sin (\theta) \iota,
$$

where $\boldsymbol{\iota}=(\cos (\phi), \sin (\phi))^{t}$. In this particular case, the shallow water equations (3.47) have the more explicit form:

$$
\begin{aligned}
\partial_{t} \widetilde{h} & +\nabla_{\xi} \cdot(\widetilde{h} \widetilde{v})=0, \\
\partial_{t}(\widetilde{h} \widetilde{v}) & +\nabla_{\xi} \cdot\left(\frac{6}{5} \widetilde{h} \widetilde{v} \otimes \widetilde{v}\right)+\frac{\delta}{2 \beta} \nabla_{\xi}\left(\cos (\theta) \widetilde{h}^{2}\right)=\bar{\kappa} \frac{\delta}{\beta} \widetilde{h} \nabla_{\xi} \cdot \mathcal{H}+\frac{1}{\beta}\left(\lambda \widetilde{h} \sin (\theta) \iota-\frac{3 \widetilde{v}}{\widetilde{h}}\right) \\
& +\frac{\delta}{\beta}\left(\frac{1}{4} \nabla_{\xi} \cos (\theta)+\theta_{R} \sin (\theta)\left(k_{1}+k_{2}\right) \iota-\frac{3}{2} \theta_{R} \sin (\theta) \operatorname{diag}\left(k_{1}, k_{2}\right) \iota\right) \frac{\widetilde{h}^{2}}{2} \\
& +\lambda^{2} \sin ^{2}(\theta)\left(\iota \cdot \nabla_{\xi} \widetilde{h}\right) \iota \frac{\widetilde{h}^{4}}{15} \\
& -\lambda^{2}\left(\frac{4}{5} \sin (\theta)\left(\iota \cdot \nabla_{\xi}\right)(\iota \sin (\theta))-\nabla_{\xi} \cdot(\iota \sin (\theta)) \iota \sin (\theta)\right) \frac{\widetilde{h}^{5}}{21} .
\end{aligned}
$$

An important remark is that the direction $\iota$ and the derivatives in that direction are important in denoting the first terms, which describe the influence of a varying topography; this is precisely the steepest descent direction, which is in the intersection of the tangent plane to the surface and the plane spanned by the gravity vector $\vec{g}$ and the normal to the bottom surface $\vec{n}$. The other quantities depend only on $\theta$, the angle between the normal to the bottom surface with the vertical and the principal curvatures of the surface. All these quantities are intrinsic and do not depend on the parametrization of the surface.

4.3. A family of models. In the following, we show that we can obtain a family of models from (3.47) which has the same accuracy. In order to simplify the discussion, we work with the shallow water equations in the steepest descent coordinates (3.47). First let us note that inserting the equation

$$
\widetilde{v}=\lambda \sin (\theta) \frac{\widetilde{h}^{2}}{3} \mathbf{e}_{1}+\mathcal{O}\left(\varepsilon \theta_{R}+\beta+\delta\right)
$$


into the momentum equation of (4.5) yields, with the same accuracy and for any constant $A$,

$$
\begin{aligned}
\partial_{t}(\widetilde{h} \widetilde{v})+\nabla_{\xi} \cdot\left(\left(\frac{6}{5}\right) \widetilde{h} \widetilde{v} \otimes \widetilde{v}-\right. & \left.\left(\frac{1}{75}+\frac{A}{9}\right) \lambda^{2} \sin ^{2}(\theta) \widetilde{h}^{5} \mathbf{e}_{1} \otimes \mathbf{e}_{1}\right) \\
+\frac{\delta}{\beta} \nabla_{\xi}\left(\frac{\cos (\theta) \widetilde{h}^{2}}{2}\right)-\bar{\kappa} \frac{\delta}{\beta} h \nabla_{\xi} \mathcal{H}= & \frac{1}{\beta}\left(\left(\lambda \widetilde{h} \sin (\theta) \mathbf{e}_{1}-\frac{3 \widetilde{v}}{\widetilde{h}}\right)\right) \\
& +\frac{\delta}{\beta} \widetilde{h}^{2} \sin (\theta)\left(\begin{array}{c}
-\frac{\theta_{R}}{2}\left(\partial_{1} \theta+\sin (\theta) \partial_{2} \phi\right)+\frac{\theta_{R}-1}{8} \partial_{1} \phi \\
-\frac{1}{8}\left(\partial_{2} \theta-\theta_{R} \sin (\theta) \partial_{1} \phi\right)
\end{array}\right) \\
& -\frac{6}{35} \lambda^{2} \widetilde{h}^{5} \sin ^{2}(\theta)\left(\begin{array}{c}
\left(\theta_{R}-1\right) \tan (\theta) \partial_{1} \theta \\
\cos (\theta) \phi_{1}
\end{array}\right) \\
& -\frac{3}{175} \lambda^{2} \widetilde{h}^{5} \sin (\theta) \cos (\theta) \partial_{1} \theta \mathbf{e}_{1} .
\end{aligned}
$$

Using the same argument and the equation

$$
\widetilde{h} \widetilde{v}=\lambda \sin (\theta) \frac{\widetilde{h}^{3}}{3} \mathbf{e}_{1}-R_{2}(\widetilde{h}, \xi)+\mathcal{O}\left(\varepsilon^{2} \theta_{R}^{2}+\beta\left(\beta+\delta+\varepsilon \theta_{R}\right)+\delta\left(\alpha+\varepsilon \theta_{R}\right)\right),
$$

one can split the term $\frac{1}{\beta}\left(\lambda \widetilde{h} \sin (\theta) e_{1}-\frac{3 \widetilde{v}}{\widetilde{h}}\right)$ in two parts for any constant $B$ :

$$
\begin{aligned}
\frac{1}{\beta}\left(\lambda \widetilde{h} \sin (\theta) \mathbf{e}_{1}-\frac{3 \widetilde{v}}{\widetilde{h}}\right) & =\frac{B}{\beta}\left(\lambda \widetilde{h} \sin (\theta) e_{1}-\frac{3 \widetilde{v}}{\widetilde{h}}\right)+\frac{1-B}{\beta}\left(\lambda \widetilde{h} \sin (\theta) e_{1}-\frac{3 \widetilde{v}}{\widetilde{h}}\right) \\
& =\frac{B}{\beta}\left(\lambda \widetilde{h} \sin (\theta) \mathbf{e}_{1}-\frac{3 \widetilde{v}}{\widetilde{h}}\right)+\frac{3(1-B)}{\beta \widetilde{h}^{2}} R_{2}(\widetilde{h}, \xi)+\mathcal{O}(\varepsilon) .
\end{aligned}
$$

The term $\frac{3(1-B)}{\beta \tilde{h}^{2}} R_{2}(\tilde{h}, \xi)$ is $\mathcal{O}(1)$ since $R_{2}(\tilde{h}, \xi)$ is $\mathcal{O}(\varepsilon)$. If we choose $B=0$, we can completely eliminate the stiff source term from (4.5), but in that case, we lose the information that $\widetilde{v}=\lambda \sin (\theta) \frac{\widetilde{h}^{2}}{3} \mathbf{e}_{1}+($ h.o.t $)$ and the fact that this asymptotic analysis was done in a quasi-stationary regime.

4.4. Lubrication models. In this section, we propose a family of models with a single equation for the variable $\widetilde{h}$, the velocity $\widetilde{v}$ being determined by $\widetilde{h}$. This kind of model is frequently used in lubrication theory, such as Benney's equation or the Kuramoto-Sivashinky equation obtained in the limit of small amplitude waves (see $[5,6]$ for more details). The validity of these equations is limited because of the limit on the amplitude of waves. Nevertheless, they are quite useful as a first approximation of the shallow water equations and are more manageable from the numerical point of view. In the literature, they are directly derived from the Navier-Stokes equations or appear as a by-product of the derivation of shallow water equations: see [11], [5, 6] for more details. We proceed here in a similar way to obtain a hierarchy of models for the fluid height $h$.

The first model equation, valid up to a first order term in $\varepsilon$, is obtained by inserting the equation

$$
\widetilde{v}=-\lambda \cos (\theta) \frac{\widetilde{h}^{2}}{3}\left(\partial_{\xi} x\right)^{-1} \mathbf{s}+\mathcal{O}\left(\varepsilon \theta_{R}+\beta+\delta\right)
$$

into the "exact" mass conservation law

$$
\partial_{t} \widetilde{h}+\nabla_{\xi} \cdot(\widetilde{h} \widetilde{v})=0
$$


Dropping small terms, one finds the conservation law

$$
\partial_{t} \widetilde{h}-\nabla_{\xi} \cdot\left(\lambda \cos (\theta) \frac{\widetilde{h}^{3}}{3}\left(\partial_{\xi} x\right)^{-1} \mathbf{s}\right)=0
$$

In the "steepest descent" coordinate system, Equation (4.13) reads:

$$
\partial_{t} \widetilde{h}+\partial_{1}\left(\lambda \sin (\theta) \frac{\widetilde{h}^{3}}{3}\right)=0
$$

An important remark is that, in the steepest descent coordinates, the flow is almost one dimensional; this makes easier the numerical simulations of the flow in that setting. The equation obtained is nothing but a Burgers equation which exhibits singularities in finite time; in order to get a more accurate model, let us replace $\widetilde{v}$ by its asymptotic expansion up to order one. For the sake of simplicity, we write the model in the "steepest descent" coordinate system. From the momentum equation (3.47) and (4.13), we deduce the order-one lubrication model

$$
\begin{aligned}
\partial_{t} \widetilde{h}+ & \nabla_{\xi} \cdot(\widetilde{h} \widetilde{v})=0, \text { with } \\
\widetilde{h} \widetilde{v}= & \frac{\lambda \widetilde{h}^{3}}{3} \sin (\theta) \mathbf{e}_{1}+\frac{\delta \widetilde{h}^{4}}{3} \sin (\theta)\left(\begin{array}{c}
\frac{\theta_{R}-1}{8} \partial_{1} \theta-\frac{\theta_{R}}{2}\left(\partial_{1} \theta+\sin (\theta) \partial_{2} \phi\right) \\
\frac{\theta_{R}}{8} \sin (\theta) \partial_{1} \phi-\frac{1}{8} \partial_{2} \theta
\end{array}\right) \\
& -\frac{\delta \widetilde{h}^{2}}{6} \nabla_{\xi}\left(\cos (\theta) \widetilde{h}^{2}\right)+\bar{\kappa} \delta \frac{\widetilde{h}^{3}}{3} \nabla_{\xi} \mathcal{H}-\frac{2 \beta \lambda^{2} \widetilde{h}^{7}}{35} \sin ^{2}(\theta)\left(\begin{array}{c}
\left(\theta_{R}-1\right) \tan (\theta) \partial_{1} \theta \\
\cos (\theta) \partial_{1} \phi
\end{array}\right) \\
& +\frac{2 \beta \lambda^{2} \widetilde{h}^{6}}{15}\left(\frac{2 \widetilde{h}}{21} \sin (2 \theta) \partial_{1} \theta+\sin ^{2}(\theta) \partial_{1} \widetilde{h}\right) \mathbf{e}_{1} .
\end{aligned}
$$

It is hard to see the effects of the curvature of the topography and those of the capillarity in this case; let us consider two cases where the lubrication equation has a simpler form. In the one dimensional case and for $\theta_{R}=1$, one finds the lubrication model

$$
\begin{aligned}
& \partial_{t} \widetilde{h}+\partial_{\xi}\left(\frac{\lambda \widetilde{h}^{3} \sin (\theta)}{3}-\frac{\bar{\kappa} \delta \widetilde{h}^{3}}{3} \partial_{\xi \xi}^{2} \theta+\frac{4 \lambda^{2} \widetilde{h}^{7}}{315} \sin (2 \theta) \partial_{\xi} \theta\right) \\
= & \partial_{\xi}\left(\frac{\widetilde{h}^{3}}{3}\left(\delta \cos (\theta)-\frac{2 \beta \lambda^{2}}{5} \sin ^{2}(\theta) \widetilde{h}^{3}\right) \partial_{\xi} \widetilde{h}\right) .
\end{aligned}
$$

In that case and up to order one, the main effects of capillarity and the curvature of the topography is to introduce a corrective term in the advection term of the equation. The dissipative term is completely similar to the dissipative term in the Benney's equation obtained for flat bottom.

When the curvature of the bottom surface is $\mathcal{O}(\varepsilon)$, let $\theta_{R}=\varepsilon \widetilde{\theta}_{R}$; the lubrication 
model reads

$$
\begin{aligned}
& \partial_{t} \widetilde{h}+\partial_{1}\left(\frac{\lambda \widetilde{h}^{3}}{3} \sin (\theta)\right) \\
= & \delta \nabla_{\xi} \cdot\left(\frac{\widetilde{h}^{4}}{8} \nabla_{\xi}(\cos (\theta))+\frac{\widetilde{h}^{3}}{3} \cos (\theta) \nabla_{\xi} \widetilde{h}\right) \\
& -\widetilde{\kappa} \delta \nabla_{\xi} \cdot\left(\frac{\widetilde{h}^{3}}{3} \nabla_{\xi}\left(\widetilde{\theta}_{R} H_{b}+\frac{1}{2} \Delta_{\xi} \widetilde{h}\right)\right) \\
& -\frac{2 \beta \lambda^{2}}{15} \partial_{1}\left(\frac{2 \widetilde{h}^{7}}{21} \sin (2 \theta) \partial_{1} \theta+\sin ^{2}(\theta) \widetilde{h}^{6} \partial_{1} \widetilde{h}+\frac{6 \widetilde{h}^{7}}{35} \sin ^{2}(\theta) \tan (\theta) \partial_{1} \theta\right) \\
& +\frac{2 \beta \lambda^{2}}{35} \partial_{2}\left(\widetilde{h}^{7} \sin ^{2}(\theta) \cos (\theta) \partial_{1} \phi\right) .
\end{aligned}
$$

In this case the dispersive effects of the capillarity appear through the term

$$
-\widetilde{\kappa} \delta \nabla_{\xi} \cdot\left(\frac{\widetilde{h}^{3}}{6} \nabla_{\xi} \Delta_{\xi} \widetilde{h}\right)
$$

\section{Conclusion}

In this paper, we have investigated systematically the slow motion of a relatively thin layer of fluid over an arbitrary topography. From the Navier-Stokes equations to Benney's equation, there is a wide range of models for shallow water flows. Numerical simulation of the full Navier-Stokes equations is cumbersome owing to the presence of the free boundary and does not give good insight into the dynamic of the fluid layer. On the other hand, Benney's equation is an oversimplified model for this kind of flow, only valid for small amplitude waves and it exhibits finite time singularities. We achieved a subsequent level of modeling by considering the time evolution of $h$ and the local flow rate $h v=\int_{0}^{h} v$; this yields the shallow water equations. We have obtained an evolution system for $h$ and $h v=\int_{0}^{h} V$, where $V$ is the component of the fluid speed parallel to the bottom surface in a closed form obtained through an asymptotic expansion, with respect to $\varepsilon$, of solutions of the full Navier-Stokes equations.

The existing models were derived from the full Navier-Stokes equations only for small curvatures of the bottom surface $[5,6],[9,10]$ or for a vanishing viscosity $[1,2]$ with or without capillarity. In this paper, the viscosity is not negligible and the curvature of the bottom is not necessarily small; the layer of fluid is entirely a boundary layer and in a first approximation, the velocity profile is parabolic provided that the bed slope never vanishes. Under an appropriate scaling of the equations, we justify (formally) the classical modeling assumptions made to derive shallow water equations: the dynamic of the layer is close to a local equilibrium (here a Nusselt flow) and the pressure is hydrostatic. We derived a family of new shallow water systems all equivalent and accurate to order one in $\varepsilon$, the film parameter.

We followed the approach of Bouchut et al. in order to give models independent of the parametrization of the bottom surface. The shallow water system obtained is written in conservative form; it has a hyperbolic part, and a stiff source term involving viscosity effects and gravity forces. The terms in front of $\bar{\kappa}$ represent the capillary effects, whereas the terms in front of $\theta_{R}$ are due to the curvature of the bottom surface and take into account centrifugal forces. The term involving $\widetilde{h}^{4}, \widetilde{h}^{5}$ are correction terms coming from the interaction between the global boundary layer 
and the model of friction at the bottom (here a no-slip condition). The stiff source term relaxes the system to the quasi-stationary Nusselt type flow.

As a first approximation the local average speed points in the steepest descent direction. Hence it is natural to write the shallow water equations for a particular parametrization of the bottom surface, the so called "steepest descent" curvilinear coordinates. As a by-product of the analysis, we obtain lubrication models valid for arbitrary topographies. Depending on the characteristic size of the bottom curvature, the capillary terms have different effects; in the case of a large curvature, the capillary force has the form $-\kappa \nabla_{\xi} \mathcal{H}_{b} h$ : it is a classical forcing term linear in $h$ : it has no dispersive effects. In order to recover the classical dispersive effects of the capillary forces, one has to assume that the bottom curvature is small; this yields capillary terms with third spatial derivative of $h$.

To finish, let us outline some prospects for this work: first we need to make some numerical simulations for the models obtained and compare the results to the one obtained with a direct simulation of the full Navier-Stokes equations. The approach proposed in this paper is only formal. It seems important that this approach should be justified mathematically even in simple situations: for that purpose, uniform estimates with respect to $\varepsilon$ of the solutions of the Navier-Stokes equations in the neighborhood of Nusselt flows are needed. Finally a mathematical analysis of the nonlinear waves for the shallow water equations obtained in this paper should give good insight into the slow dynamics of shallow water flows.

\section{REFERENCES}

[1] F. Bouchut, A. Mangeney-Castelnau, B. Perthame and J.-P. Vilotte, A new model of Saint Venant and Savage-Hutter type for gravity driven shallow water flow, C.R. Acad. Sci. Paris, série I, 336, 531-536, 2003.

[2] F. Bouchut and M. Westdickenberg, Gravity driven shallow water models for arbitrary topography, Commun. Math. Sci., 2(3), 359-389, 2004.

[3] D. Bresch and B. Desjardins, Existence of global weak solutions for a 2D viscous shallow water equations and convergence to the quasi-geostrophic model, Comm. Math. Phys., 238(1-2), 211-223, 2003.

[4] D. Bresch, B. Desjardins and C.-K. Lin, On some compressible fluid models: Korteweg, lubrication, and shallow water systems, Comm. Partial Differential Equations, 28(3-4), 843-868, 2003.

[5] C. Ruyer-Quil and P. Manneville, Modeling film flows down inclined planes, Eur. Phys. J. B, 6, 277-298, 1998.

[6] C. Ruyer-Quil and P. Manneville, Improved modeling of flows down inclined planes, Eur. Phys. J. B, 15, 357-369, 2000.

[7] J.-F. Gerbeau and B. Perthame, Derivation of viscous Saint-Venant system for laminar shallow water; numerical validation, Discrete Contin. Dyn. Syst. Ser. B, 1(1), 89-102, 2001.

[8] A. Oron, S.H. Davis and S.G. Bankoff, Long-scale evolution of thin fluid film, Rev. Mod. Phys., 69, 931-980, 1997.

[9] R. Valery Roy, A.J. Roberts and M.E. Simpson, A lubrication model of coating flows over a curved substrate in space, J. Fluid. Mech., 454, 235-261, 2002.

[10] A.J. Roberts and Zhenquan Li, An accurate and comprehensive model of thin fluid flows with inertia on curved substrate, J. Fluid. Mech., 553, 33-73, 2006.

[11] V.Y Shkadov, Wave conditions in the flow of a thin layer of a viscous liquid under the action of gravity, Izv. Ak. Nauk SSSR, Mekh. Zhi. Gaza, 2, 43-51, 1967.

[12] B. Gjevik, Occurrence of finite-amplitude surface waves of falling liquid films, Phys. Fluids, 13, 1918, 1970.

[13] A. Pumir, P. Manneville and Y. Pomeau, On solitary waves running down on inclined plane J. Fluid Mech., 135, 27-50, 1983.

[14] J.-P. Vila, Two-moment closure equations of shallow water type for thin film gravity-driven laminar flow gravity driven, in preparation. 